\title{
Filament Eruption Driving EUV Loop Contraction then Expansion above a Stable Filament
}

\author{
Ramesh Chandra ${ }^{1}$, Pascal Démoulin ${ }^{2,3}$, Pooja Devi ${ }^{1}$, Reetika Joshi ${ }^{1}$, Brigitte Schmieder ${ }^{2,4,5}$ \\ ${ }^{1}$ Department of Physics, DSB Campus, Kumaun University, Nainital-263 001, India \\ rchandra.ntl@gmail.com \\ ${ }^{2}$ LESIA, Observatoire de Paris, Université PSL, CNRS, Sorbonne Université, Université de Paris, \\ 5 place Jules Janssen, 92195 Meudon, France \\ ${ }^{3}$ Laboratoire Cogitamus, 1 3/4 rue Descartes, 75005 Paris, France \\ ${ }^{4}$ Centre for Mathematical Plasma Astrophysics, Department of Mathematics, KU Leuven, 3001 \\ Leuven, Belgium \\ ${ }^{5}$ LSUPA, School of Physics and Astronomy, University of Glasgow, Scotland
}

\begin{abstract}
We analyze the observations of EUV loop evolution associated with the filament eruption located at the border of an active region. The event SOL2013-03-16T14:00 was observed with a large difference of view point by the Solar Dynamics Observatory and Solar Terrestrial Relations Observatory -A spacecraft. The filament height is fitted with the sum of a linear and exponential function. These two phases point to different physical mechanisms such as: tether-cutting reconnection and a magnetic instability. While no X-ray emission is reported, this event presents the classical eruption features like: separation of double ribbons and the growth of flare loops. We report the migration of the southern foot of the erupting filament flux rope due to the interchange reconnection with encountered magnetic loops of a neighbouring AR. Parallel to the erupting filament, a stable filament remains in the core of active region. The specificity of this eruption is that coronal loops, located above the nearly joining ends of the two filaments, first contract in phase, then expand and reach a new stable configuration close to the one present at the eruption onset. Both contraction and expansion phases last around $20 \mathrm{~min}$. The main difference with previous cases is that the PIL bent about $180^{\circ}$ around the end of the erupting filament because the magnetic configuration is at least tri-polar. These observations are challenging for models which interpreted previous cases of loop contraction within a bipolar configuration. New simulations are required to broaden the complexity of the configurations studied.
\end{abstract}

Subject headings: Sun: Filament - Sun: Flares - Sun: Loop 


\section{Introduction}

Solar filaments (or prominences, when observed at solar limb) are cool and dense material plasma suspended in a million degree hot corona (Mackay et al. 2010; Labrosse et al.2010; Parenti 2014). They are located above the photospheric inversion line (PIL) of the vertical component of the magnetic field. They are observed in the active as well as in the quiet solar atmosphere or between two active regions. Since the plasma of filaments is about a factor one hundred denser than the coronal plasma it needs to be supported by a force against the action of gravity. The

existence of stable support in magnetic dips was initially proposed by Kippenhahn \& Schlüter (1957). Present models typically involved a magnetic structure with the filament plasma caught in magnetic dips (see the review by Mackay et al. 2010). Magnetic dips can be present naturally in potential fields with a quadrupolar magnetic configuration but not with a bipolar configuration. Several models have been developed such as the sheared arcade model (Antiochos et al.|1994), and the flux rope (FR) model (Aulanier \& Démoulin 1998; Priest et al. 1989; Aulanier \& Schmieder 2002). Finally, the existence and the evolution of a filament is strongly linked to the associated magnetic field configuration.

Usually, at some point of their evolution filaments become unstable and erupt. The eruption can be confined by the overlying magnetic field, then is called a failed eruption. In the opposite case, the filament, the surrounding coronal magnetic field and the plasma become a coronal mass ejection (CME) which is ejected towards the interplanetary medium (e.g., Gibson \& Fan 2006, Chandra et al. 2010; Schmieder et al. 2013; Kliem et al. 2014). Therefore filament eruptions are thought to play a diagnostic role for the origin of CMEs because of their much higher plasma density than the surrounding corona. Eruptions and associated phenomena, such as the formation of flare ribbons and their separation with time, were initially explained by the CSHKP model (Carmichael 1964; Sturrock 1966; Hirayama 1974; Kopp \& Pneuman 1976). Later on, this 2dimension model was extended into a 3-dimensional model that could explain eruption associated phenomena including slipping reconnection, circular ribbon formation, and the magnetic shear evolution of flare loops (Aulanier et al. 2010; Janvier et al.2015).

Different mechanisms could explain the trigger of eruptions, which include the magnetic breakout (Antiochos et al. 1999), tether cutting (Moore \& Sterling 2006), kink instability (Török \& Kliem 2005) and the torus instability or catastrophe model (Kliem \& Török 2006). The observations and models of both the possible pre-eruptive magnetic configurations and the eruption mechanisms have recently been reviewed (Green et al. 2018; Georgoulis et al. 2019; Patsourakos et al. 2020). As the FR is erupting according to the Lin \& Forbes (2000) model, a thin current sheet (CS) is formed behind the erupting FR. The erupting FR can be ejected from the corona into the heliosphere thanks to the magnetic reconnection occurring in the current sheet behind the FR. This reconnection transforms part of the stabilizing magnetic connections passing above the FR 
to connections located below the FR (flare loops) and to connections wrapped around the FR, so further building the FR.

One of the remarkable phenomena accompanying solar eruptions is the evolution of coronal loops. This phenomenon is reported with various space borne observations in flare loops with the shrinkage of individual loops while the global loop system expand upwards as further flare loops are formed (Forbes \& Acton 1996; Li \& Gan 2006; Sui et al. 2004; Zhou et al. 2008; Joshi et al.2009). Moreover, since the launch of SDO in 2010, observational evidences of coronal loop contraction and expansion, not associated to flare loops, are increasing (Sun et al. 2012; Gosain 2012; Liu et al. 2012; Zhou et al. 2013; Simões et al. 2013; Shen et al. 2014; Dudík et al. 2017; Wang et al. 2018; Dudík et al. 2019; Devi et al. 2021). This is a consequence of SDO continuous high temporal and spatial resolution observations. This phenomena is usually observed together with eruptive solar flares. These loops are already present before the flare, so they are different from flare loops which are formed by reconnection during the flare. The speed of the contraction and expansion is typically between a few $\mathrm{km} \mathrm{s}^{-1}$ and $40 \mathrm{~km} \mathrm{~s}^{-1}$. Furthermore, in some cases the contracted loops end up oscillating (e.g., Gosain 2012; Liu et al. 2012; Simões et al. 2013).

To explain the phenomena of the loop contraction two mechanisms were explored. The first mechanism is the "magnetic implosion" conjecture proposed by Hudson (2000). According to this the energy generated during a solar eruption has its origin in the implosion of the magnetic configuration in a nearby part of the corona. The expansion followed by contraction in coronal loops is recently simulated in 3D by Wang et al. (2021). The physical interpretation of such implosion has later changed. For example, Simões et al. (2013) interpret the observed loop contraction with an implosion which is a consequence, and not a driver, of the energy release during the event. Later on, Russell et al. (2015) interpret the loop contraction as the consequence of the reduction of the magnetic pressure due to the magnetic reconnection occurring in the flare. Several observations of loop contraction are interpreted as supporting this mechanism (Wang et al. 2018). The second mechanism is proposed by Aulanier et al. (2010) and Zuccarello et al. (2017). This model is based on the 3D magnetohydrodynamics (MHD) numerical simulations of the formation then eruption of a FR in a bipolar magnetic configuration modelling an AR configuration. According to their model, the loop contraction/expansion is the result of the vortex forming on both sides of erupting FR legs. These vortex flows drive the coronal loops and as a result of this the contraction/expansion in loops observed, depending where the loops are in the vortex. The careful analysis of the contraction/expansion of loops in some studied eruptions filament support the conclusion of the MHD simulations (Dudík et al. 2017, 2019; Devi et al. 2021).

Coronal loops can also be excited by propagating coronal waves, in particular fast magnetoacoustic waves (Wills-Davey \& Thompson 1999; Ballai 2007; Ballai et al. 2008). These waves can be generated by sudden energy release process like the phenomena of solar flares and CMEs. 
The propagation of coronal waves can be considered as a freely propagating wavefront which is observed to interact with coronal loops (see, e.g. Wills-Davey \& Thompson 1999; Ballai et al. 2008).

In this paper, we study loop contraction and expansion associated with a filament eruption on 2013 March 16 observed in EUV wavelengths by the AIA imager aboard SDO and by EUVI aboard STEREO with a large difference of viewing points. The specificity of this event is that the loop contraction and expansion were occurring not only in coronal loops located nearby the end point of the erupting filament, but also in loops located over a stable filament. This filament is located on the side of the erupting one with a nearly parallel orientation of most parts of both filaments, which are also nearly joining at one end. Then, this is an interesting eruption to study as it broaden the range of configurations where the loop contraction and expansion could occur. The layout of the paper is as follows: Section 2 describes the data sets, the morphology of filament eruption, the history of the magnetic configuration of the involved active region (AR), the kinematics of the eruption, and the observational results related to EUV loop contraction and expansion. The physical interpretation of the obtained results is given in Section 3. Finally in Section 4, the conclusion of the study is given.

\section{Observations}

\subsection{Overview of the Event}

The filament eruption on 2013 March 16 was observed by the Atmospheric Imaging Assembly (AIA, Lemen et al. 2012) onboard Solar Dynamics Observatory (SDO, Pesnell et al. 2012) at different EUV wavelengths with pixels of $0.6^{\prime \prime}$ and cadence of $12 \mathrm{~s}$. For our present analysis, we used the AIA data of 304, 171, 193 and $211 \AA$ which correspond to the wavelengths where the eruption is observed the best. For the magnetic configuration of the filament region, we analyzed the photospheric magnetic field line-of-sight data of Helioseismic Magnetic Imager (HMI, Schou et al. 2012). The pixel resolution and the temporal resolution of the HMI magnetic field data is 0.5 " and 45 s respectively. The eruption was also observed by the Solar Terrestrial Relations Observatory Ahead (STEREO A, Howard et al.2008) spacecraft with a cadence of $10 \mathrm{~min}$. All the above mentioned data are processed by the solar soft package. To remove the solar rotation, we rotated all used AIA analyzed images at 12:30 UT. For this purpose, we used the "drot_map" routine available in solar soft. In order to enhance the contrast of the AIA data, we processed the images with the Multi-Gaussian Normalization (MGN) method developed by Morgan \& Druckmüller (2014). To further outline the evolution we create base-difference movies, both with and without MGN method, with the base time set at 12:30 UT. 


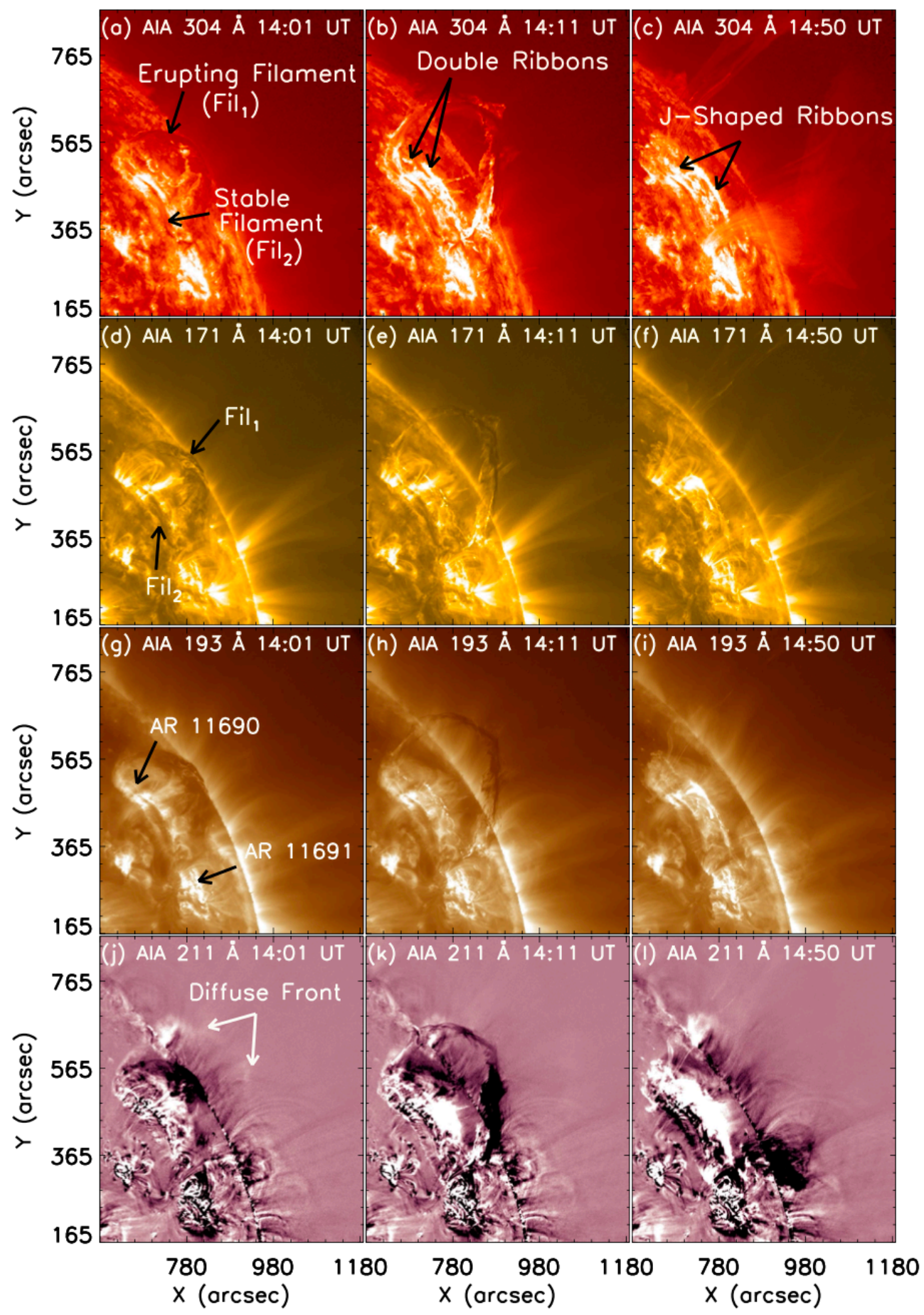

Fig. 1.- Multi-wavelength view of the filament eruption of 2013 March 16 in AIA 304, 171, 193 and $211 \AA \AA$. The AIA $211 \AA$ A images are base difference images (base time: 12:30 UT). The location of the erupting filament $\left(\mathrm{Fil}_{1}\right)$, the stable filament $\left(\mathrm{Fil}_{2}\right)$ and the flare ribbons are indicated in panels (a), (d) and (b, c), respectively. The AR involved in the eruption, AR 11690, and the southern one are indicated in panel (g). The propagating diffuse front above the filament is shown in panel (j). Movie of these data is available in the Electronic Supplementary Materials. The animation starts at 12:30 UT and end at 15:00 UT. The realtime duration of the animation is 25 seconds. 


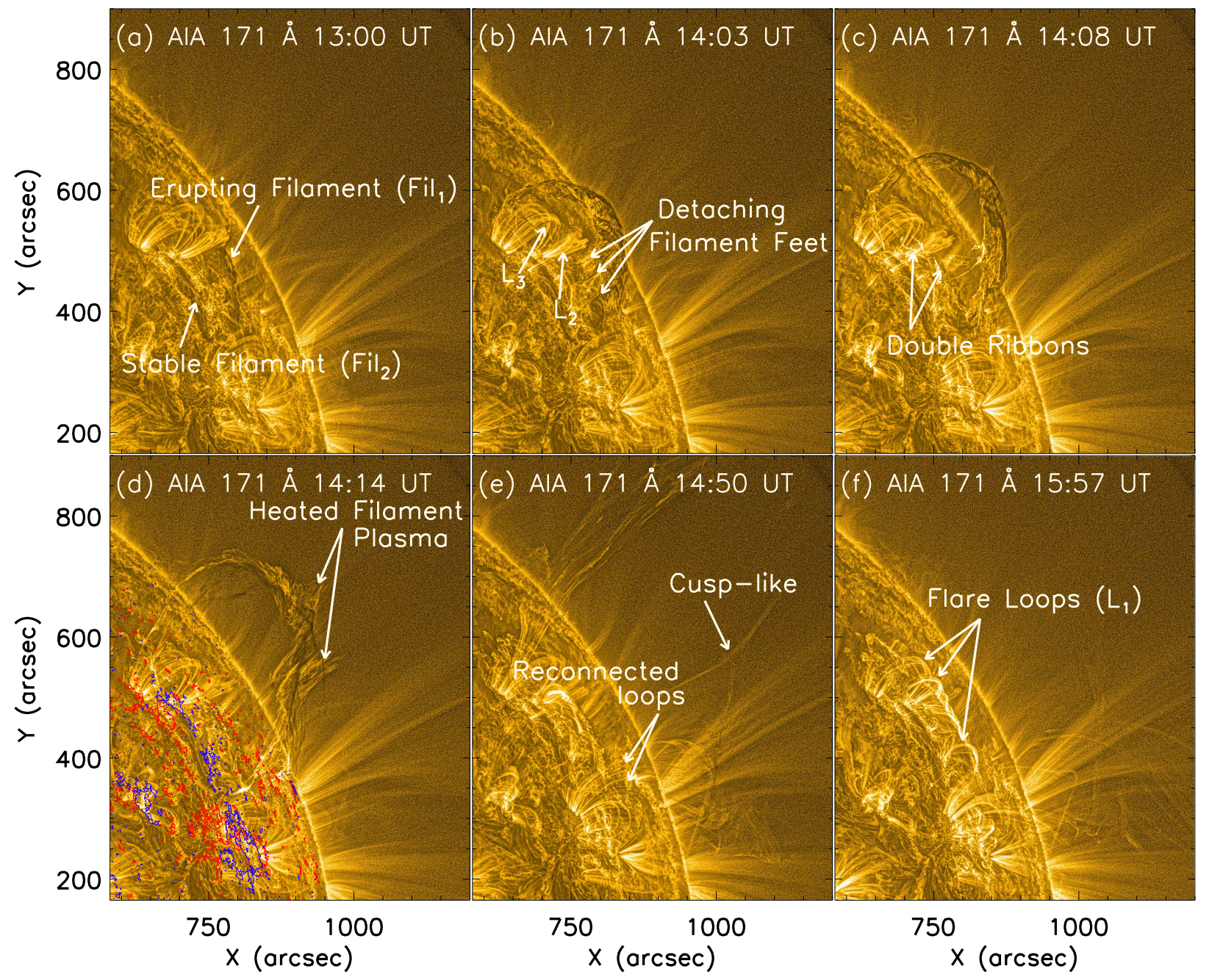

Fig. 2.- Evolution of the filament eruption with AIA images at $171 \AA$ processed with the MGN method (Section 2.1). The erupting (Fil $)_{1}$ and the stable $\left(\mathrm{Fil}_{2}\right)$ filaments are indicated in panel (a). Specific characteristics of the erupting filament are pointed in panels (b) and (d). The consequences of the main energy release are indicated in panels (c) and (f) with flare ribbons and flare loops $\left(\mathrm{L}_{1}\right)$, respectively. The HMI positive/negative polarity contours are overlaid in panel (d) with red/blue contours (contour level: $\pm 30 \mathrm{G}$ ). The loop systems $\mathrm{L}_{2}$ and $\mathrm{L}_{3}$, located above $\mathrm{Fil}_{2}$, are indicated in panel (b). The reconnected loops, marked in panel (e), are due to the reconnection of the southern leg of the erupting FR with the magnetic field of AR 11691 (located southward of AR 11690, see Figure 1 $\mathrm{g}$ ). The associated movie is available in the Electronic Supplementary Materials. The animation starts at 12:30 UT and end at 15:00 UT. The realtime duration of the animation is 25 seconds. 
The erupting filament $\left(\mathrm{Fil}_{1}\right)$ on 2013 March 16 was located at the north-west border of the NOAA AR 11690 (Figure 1 g). A second stable filament $\left(\mathrm{Fil}_{2}\right)$ is present on the main PIL of AR 11690. Both filaments are indicated with arrows in Figure 1 1 a,d. The evolution of the erupting filament Fil $_{1}$ in AIA 304, 171, 193 and $211 \AA$ is summarized with three selected times in Figure 1 . The accompanying movies provide a detailed view of the eruption. The filament started to move upwards at $\approx 13: 19$ UT in the north-west direction (best seen with base-difference movies). Much later on, at about 13:59 UT, two flare ribbons started to develop below the erupting filament. The double ribbons, indicated by arrows in Figure $1 \mathrm{p}$, are well observed in 304, 171, 193, and $211 \AA$. As the filament was moving upward, the double ribbons separated from each other as predicted in the standard flare model (Section 1). Furthermore, J-shaped ribbons are present after 14:29 UT which indicate the eruption of a FR (Démoulin et al. 1996; Aulanier et al. 2010). These ribbons are best seen in the base-difference of AIA $304 \AA$.

The MGN technique is applied to AIA data in order to enhance the coronal structures so that the morphology and the dynamics of the eruption could be better understood. Figure 2 shows the evolution with the MGN technique applied to $171 \AA$. Arrows point to the main observational features. The filament eruption is observed the best with the $171 \AA$ filter with more contrasted images which reveal both the EUV absorption by the dense and cold filament plasma and the emission of heated plasma (e.g., part of the filament, flare ribbons and loops).

On mid 2013 March 16 the filament Fil $1_{1}$ erupted as a coherent structure. This is emphasized by a sharp leading edge moving as a coherent entity (Figure $2 \mathrm{a}-\mathrm{c}$ ). At the beginning of the filament eruption three dark features are extending below the filament body. They are pointed by three arrows in Figure 2b. These extensions are filament feet/barbs which have been identified previously mostly in quiescent filaments (Aulanier et al. 1999; Mackay et al. 2010). As the eruption progresses, the feet of the filaments become more clearly visible, before getting split with the lower part falling towards the chromosphere and with the upper part being integrated in the eruptive configuration. During this splitting, the associated plasma is changing from absorption to emission, so it is heated. They are both indications that magnetic reconnection occurs below the erupting FR.

As the filament erupts, its two ends stay anchored at low heights (Figure $2 \mathrm{a}-\mathrm{d}$ ). This allows to identify the two footpoints of the erupting FR which are located in the northward and westsouthward periphery of AR 11690, in negative and positive photospheric polarities, respectively (Figure 3d). Later on in the eruption, a cusp-like shape appears in emission in $171 \AA$ (Figure 22). However, the temporal evolution is more compatible with the crossing in projection of two loops (see associated movie).

The evolution of the southern part of the erupting filament is complex (see attached movies in 304, 171, 193 and $211 \AA$ ). We interpret this evolution as the reconfiguration of the southern FR leg. During its upward ejection, the FR expands and reconnects, at least partially, with the main 
bipolar field of AR 11691. This shifts the FR magnetic anchorage from the positive polarity in front of AR 11690 to the positive following polarity of AR 11691 as traced by the new connections

pointed in Figure 2e (with "Reconnected Loops"). A part of the dense filament plasma fall along and fill partly these new connections as seen the best in 171 and $304 \AA$ base-difference movies. We conclude that the lateral drift of the filament foot is accomplished via interchange reconnection with encountered southern magnetic loops. These observations are globally consistence with the previously reported observations of such lateral drift of the foot of an erupted prominence/filament (Hori 2000; van Driel-Gesztelyi et al. 2014; Dudík et al. 2019; Lörinčík et al. 2019; Zemanová et al. 2019). Another part of the erupted filament drains to the end region of the J-shaped flare ribbon in the west (towards polarity $P_{0}$ in Figure 3), which is near the periphery of the flux rope leg. This is best seen in AIA higher temperature, e.g. 211 and $193 \AA$, and it implies that the magnetic configuration of this filament leg splits.

The flare associated to this filament eruption is weak as it does not saturate the EUV detectors. Indeed the GOES instrument does not observe any flux enhancement in X-rays, then this event is not classified as an X-ray flare. Still, it presents all the EUV characteristics of a two ribbons flare with two separating ribbons and an arcade of flare loops, $\mathrm{L}_{1}$, linking them (Figures $2 \mathrm{r}, \mathrm{f}$ ). Both these ribbons and flare loops are partly hidden by other sets of loops, $\mathrm{L}_{2}$ and $\mathrm{L}_{3}$, located in front in the AIA images (Figure $2 \mathrm{~b}$ ). These $\mathrm{L}_{2}$ and $\mathrm{L}_{3}$ sets are not part of the flare which is associated to $\mathrm{Fil}_{1}$ eruption. Rather these $\mathrm{L}_{2}$ and $\mathrm{L}_{3}$ sets are located over the stable filament $\mathrm{Fil}_{2}$, and they are studied in Section 2.4.

\subsection{Magnetic Configuration}

The evolution between 2013 March 11 - 16 of the magnetic field in the vicinity of the filaments is displayed in Figure 3 in the local solar frame. The main magnetic polarities are labeled in panel (e). AR 11690 is mostly in a decaying stage, with the emergence of a bipole in its negative polarity $\mathrm{N}_{1}$ (Figure $3 \mathrm{~b}$-d). This induced a reorganization of its leading polarity from 12 to 14 March. More importantly, long-term small scale cancellations of the magnetic flux are observed at the PIL between $\mathrm{N}_{1}$ and $\mathrm{P}_{0}$ (this is best seen in the associate movie). These cancelations are induced by the dispersion of the magnetic polarities due to sub-photospheric convection motions with a time scale of days. This flux cancellation is expected to build the FR which supports the filament (van Ballegooijen \& Martens 1989). It is also at the origin of the slow evolution of the magnetic configuration which can bring it to instability (Amari et al.2010; Aulanier et al. 2010).

The erupting filament $\mathrm{Fil}_{1}$ is located at north-west side of the AR 11690 as shown with the red contour of $\mathrm{H} \alpha$ observation co-aligned with the magnetogram (Figure $3 \mathrm{~d}$ ). More precisely the bottom of the $\mathrm{H} \alpha$ filament is following the PIL between the AR negative leading polarity $\mathrm{N}_{1}$ and 


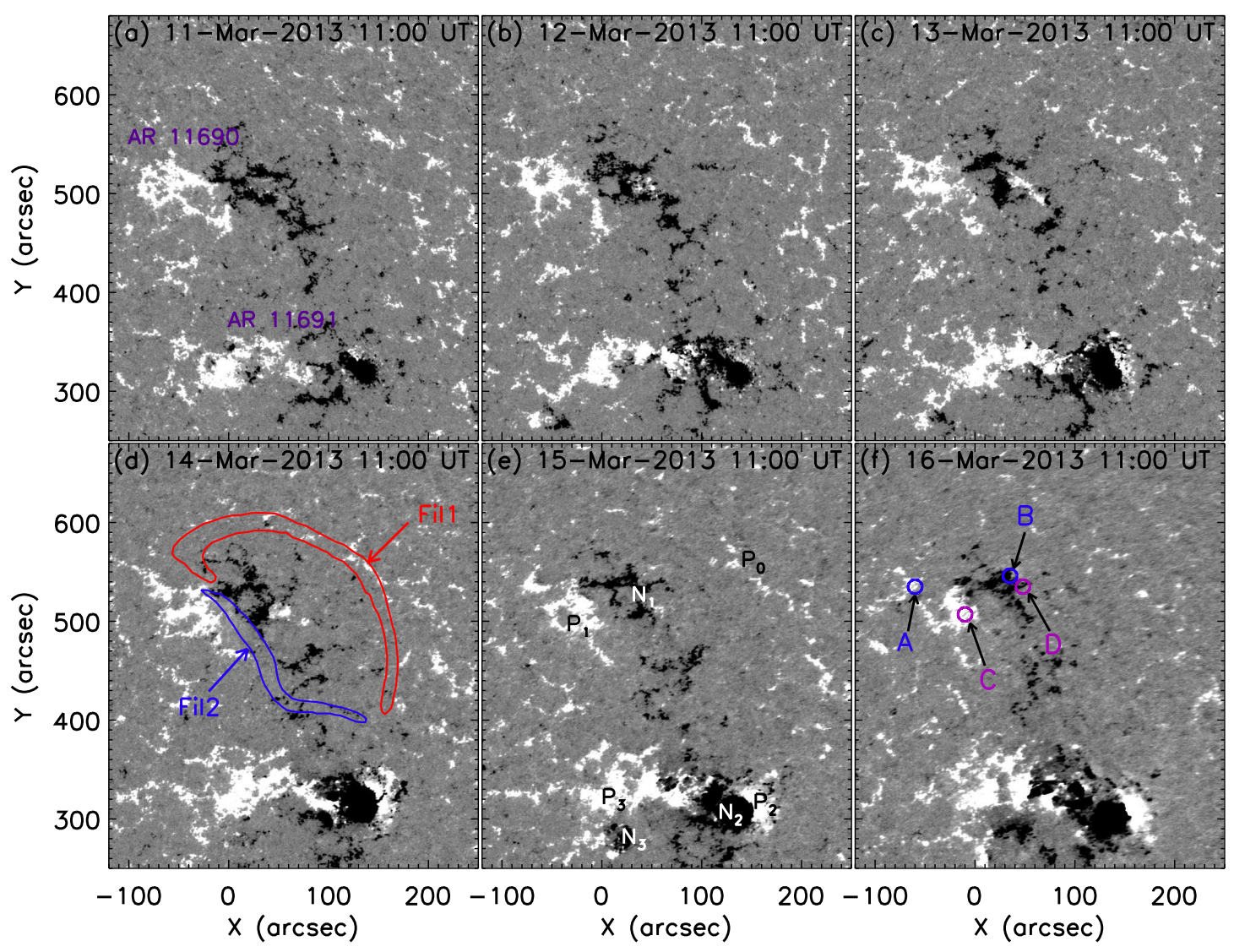

Fig. 3.- Evolution of the longitudinal component of the photospheric magnetic field the ARs 11690 and 11691. The observations are derotated to the central meridian location. The contours in panel (d) delimitate the location of the two filaments observed in $\mathrm{H} \alpha$. The red contour indicates the erupting filament $\mathrm{Fil}_{1}$, while the blue contour indicates the stable filament $\mathrm{Fil}_{2}$. The different magnetic polarities are named by $P_{0}$ (remnant field of a previous AR), $P_{1}, N_{1}$ (AR 11690), $P_{2}, N_{2}, P_{3}, N_{3}$ (AR 11691). The locations of the loop foot-points, defined in Figure 6 $\mathrm{b}$, are indicated by the pairs A, B and C, D, respectively in panel (f). An associated movie from 2013 March 11 00:00 UT to 2013 March 16 17:00 UT of realtime duration 22 seconds is available in the Electronic Supplementary Materials. 
a dispersed large-scale positive polarity $\mathrm{P}_{0}$ (the remnant of an earlier $\mathrm{AR}$ ). The filament extends on the positive polarity indicating that it is inclined towards the west. The explanation for the westward inclination of the filament $\mathrm{Fil}_{1}$ away from its underlying PIL is the asymmetry of the magnetic field strength on both sides of the PIL. This inclination is present in MHD simulation during the equilibrium phase and even more visible during the eruption phase (Aulanier et al. 2010; Török et al. 2011; Titov et al. 2018, 2021). From coronal observations, such asymmetry is also known to cause eruptions to be inclined toward the weak-field side (e.g., Panasenco et al. 2013; Kay et al. 2015). Next, a narrow stable filament, Fil ${ }_{2}$, is located along the main PIL within AR 11690 (blue contour in Figure 3d). The filament Fil ${ }_{2}$ is following well the local PIL and it is narrower than $\mathrm{Fil}_{1}$ as typically observed for filaments within ARs compare to filaments at the periphery of ARs. Furthermore, both filaments, Fil $_{1}$ and $\mathrm{Fil}_{2}$, are on the same PIL located around the polarity $\mathrm{N}_{1}$. Fil ${ }_{1}$ is even curving slightly inside AR 11690 in its northern part (Figure 3d).

As mentioned in Section 2.1, the shape of the observed ribbons is reverse J-shaped. Such orientation of the ribbons is an evidence of left handedness in the AR (Démoulin et al.|1996; Williams et al. 2005; Chandra et al. 2009). Based on this, we infer the handedness of Fil $_{1}$ is left-handed. Another method to determine the handedness of the filament is as follows. We have identified the magnetic polarities at the end points of filament $\mathrm{Fil}_{1}$ and found that the northern/southern footpoint are located in positive/negative polarities respectively (Figure 3 $\mathrm{d}$ ). In addition to this, the eastern/western side of the filaments are in negative/positive polarities respectively. Using these identifications, we have determine the handedness of the filament (see figure 5 of Mackay et al. 2010) and conclude that the filament $F_{1}$ has a left-handed configuration. The same method is applied for the filament $F_{2}$ which is also found to be in a left-handed configuration. However, this correspondence of handedness is not sufficient to infer that both filaments are in a single magnetic configuration along the same PIL. We found during the quiet phase, before 12:40 UT on March 16, it is difficult to find a separation between the two filaments. However, the eruption of Fil 1 and not of $\mathrm{Fil}_{2}$ is a clue supporting separated magnetic field configuration, with the limitation that filament plasma outlines only a small fraction of the magnetic configuration. Then, we will first consider below two nearly parallel filaments, before coming back in Section 3.2 to the details of this northern part of the PIL where they are nearby.

Finally, another AR, NOAA 11691, is developing southward to AR 11690 and with the same global bipole orientation $\left(\mathrm{P}_{3}-\mathrm{N}_{2}\right.$, Figure 3). Since AR 11691 is nearby AR 11690 and the erupting magnetic structure is strongly expanding southward, the coronal field of AR 11691 partly reconnects with the erupting magnetic field (see Figure 2e, related movie, and Section 2.1). 

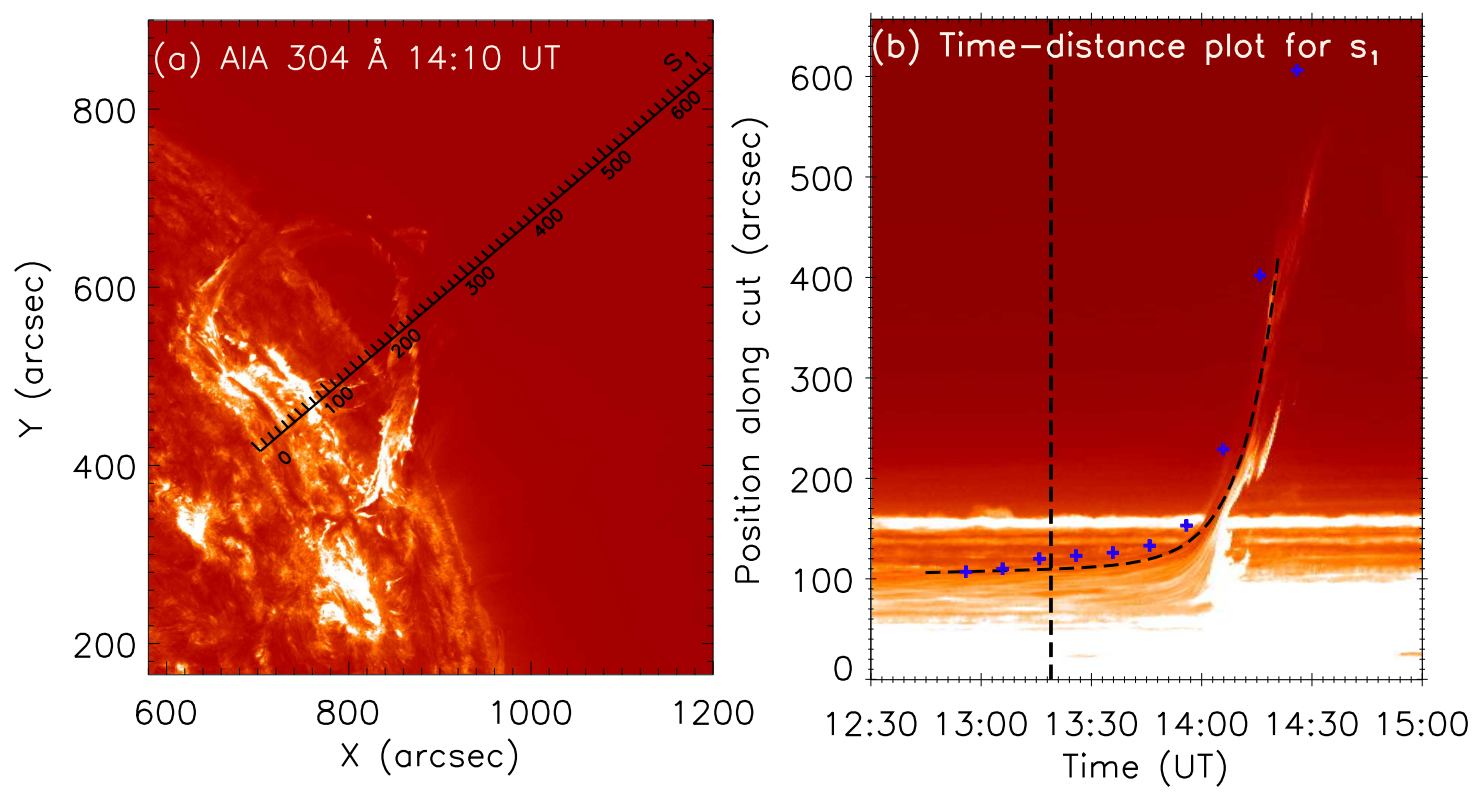

Fig. 4.- (a) AIA $304 \AA$ A image with the location of slice $\mathrm{S}_{1}$ used for the analysis of the filament Fil 1 height-time evolution. (b) Height-time plot along the slice $S_{1}$. The height is corrected from solar rotation. The vertical dashed line indicates the approximate onset time of the filament eruption. The black dashed line is the fit of Equation (1) to the filament apex as traced with the AIA 304 $\AA$ data along slice $S_{1}$. The blue crosses represent the apex height of the filament leading edge derived by triangulation with the data of SDO/AIA and STEREO A/EUVI (see Figures 1 and 5). The height is set at the same position for the first blue cross. During the eruption these heights are slightly above the AIA $304 \AA$ apex and its fit with Equation (1) due to a projection effect.

\subsection{Eruption Kinematics}

We explore the eruption kinematics using AIA data sets. A global and qualitative view of the eruption is given by the three movies attached to Figure 1. A quantitative analysis is done with the slice $S_{1}$ shown in Figure 4 a. We define the location of slice $S_{1}$ in AIA images to follow the filament leading edge. The measured height is corrected from solar rotation. While the filament eruption and its consequences are seen the best with $171 \AA$ data, the early part of the eruption is partly masked with the sets $\mathrm{L}_{2}$ and $\mathrm{L}_{3}$ of coronal loops present in front of the erupting filament (middle left of Figure 2 a, b). These loops are not emitting in $304 \AA$, then we privilege this filter to study the pre-eruption phase, as well as the whole filament eruption, while we also apply the same method to the other filters for completeness.

The kinematics of the filament eruption is an important clue for understanding the triggering mechanism of the eruption. Therefore, in order to analyze the kinematics of the filament eruption, 
we visually select points along the bright tracks of the time-distance plots as shown in Figure $4 \mathrm{p}$. Cheng et al. (2020) have analyzed 12 eruptions and test several functions to fit the data. They found that the combination of a linear and exponential time dependences provide the best description of the full data set, so a lowest $\chi^{2}$ for most eruptions, compare to previously suggested functions (like a power law of time). The most general equation analyzed by Cheng et al. (2020) is

$$
h(t)=a e^{b\left(t-t_{0}\right)}+c t+d .
$$

Here, we note that $t_{0}$ is a redundant parameter as the first term can be rewritten as $a e^{-b t_{0}} e^{b t}=a^{\prime} e^{b t}$ so that all the combinations of the three parameters $a, b, t_{0}$, which provides the same $a^{\prime}$ value, define exactly the same function. Said differently, $b$ is defined by the temporal behavior of the data while changing $a$ in Equation (1) could be exactly compensated by changing $t_{0}$. Then, we fix $t_{0}$ to a given value, 12:45 UT as Cheng et al. (2020) fixed it to their first data point. The coefficients $a, b, c$, and $d$ are determined by minimizing the reduced $\chi^{2}, \chi_{r}^{2}$, between the data and Equation (1). The fitting is done using the mpfit routine available in the solar software (SSWIDL). The goodness of the fitting is given by the reduced $\chi_{r}^{2}$ value obtained. The $\chi^{2}$ is defined by $\chi^{2}=\sum_{i=1}^{N}\left[h_{i}(t)-H_{i}(t)\right]^{2}$, where $\mathrm{h}_{i}$ and $\mathrm{H}_{i}$ are the fitting and measured heights, respectively and ' $\mathrm{t}$ ' is the time. This formula is slightly different than the one used by Cheng et al. (2020) since we are not including the error for each measured height (the minimum found assumed the same error for each measurement). Finally we calculated the reduced $\chi_{r}^{2}$ value as $\frac{\chi^{2}}{D O F}$, where DOF is the number of the degree of freedom. We find the minimum $\sqrt{\chi_{r}^{2}}=1.4^{\prime \prime}$. This is only slightly above two pixels of AIA $\left(1.2^{\prime \prime}\right)$, indicating that Equation (1) provides a close representation of the data.

During the earlier times of Figure $4 \mathrm{~b}$, a slow linear increase of the filament height is present, defined as the slow-rise phase by Cheng et al. (2020). The fit of Equation (1) to the data provides $c \approx 2 \mathrm{~km} \mathrm{~s}^{-1}$ in all AIA channels. This slow-rise phase is typically associated with the presence of weak brightenings. They are interpreted as the consequence of tether-cutting reconnection which allow the slow upward motion. Later on, during the acceleration phase, the speed of the eruption increases exponentially. The maximum speed of the eruption was computed by derivating the height-time fit of the data. The calculated maximum speed reached up to $300 \mathrm{~km} \mathrm{~s}^{-1}$ within the AIA field of view. This increase occurs on the time scale $1 / \mathrm{b} \approx 9.6 \mathrm{~min}$. This exponential behavior is characteristic of the linear development of an instability.

The beginning of the eruption is difficult to define precisely as the filament top is smoothly changing from a linear to an exponential rise. Also, there is no characteristics time defined by Equation (1) as $t_{0}$ is an ill defined parameter (see above), and then cannot be associated to an onset time. Indeed, for both a linear and exponential behaviors there is no specific time which can be referred to (said differently both function can be shifted in time while keeping the same form). Then, an extra information needs to be added to define the beginning of the eruption from Equation (1). The instability starts at least before the exponential rise is large enough to be detected. This is best 

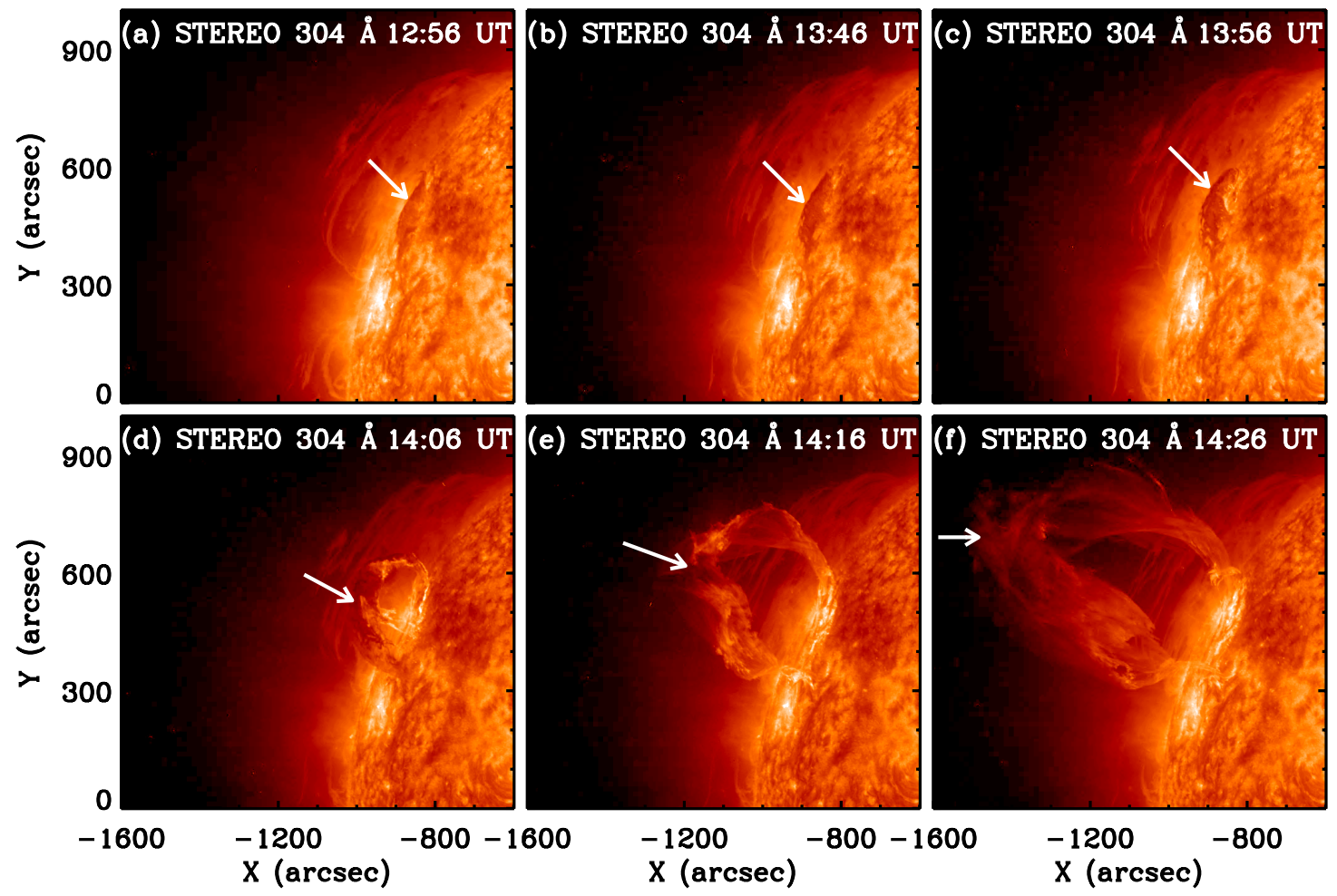

Fig. 5.- Evolution of the filament observed by STEREO A /EUVI in $304 \AA$ at the eastern solar limb. The view point is on the other side of the filament as observed with AIA (Figure $4 \mathrm{a}$ ) with a longitude difference of $130^{\circ}$ between the spacecraft. The filament/prominence leading edge apex is indicated by arrows.

quantified with the velocity, $a b e^{b t}$ becoming larger than a threshold value $v_{t}$. Then, the eruption start time is defined as:

$$
t_{\text {start }}=\frac{1}{b} \ln \left(\frac{v_{t}}{a b}\right)
$$

Taking $v_{t}=c$ sets the exponential velocity equal to the linear velocity, so the growth of the instability well visible above the linear rise. This occurs at $\approx 13: 30$ UT. The eruption is expected to starts even earlier on. The exponential is well detectable over the fluctuations of the slow-rise phase when it is larger than three times the standard deviation, which is found to be $\approx 0.62 \mathrm{~km}$ $\mathrm{s}^{-1}$. Including this velocity as $v_{t}$ in Equation (2) defines the eruption start at 13:19 UT, so $11 \mathrm{~min}$ before. This last time is expected to be closer to the real eruption start, which is expected to be even earlier on when the exponential was too small to be detectable. To be conservative, we set the starting time at 13:19 UT. 
The eruption was also well observed by the STEREO A spacecraft with a different viewing angle in longitude. The angle between the STEREO A and the SDO on 2013 March 13 was $130^{\circ}$ and for STEREO A the filament was located close to the eastern limb. The evolution of the eruption in $304 \AA$ is shown in Figure 5. The arrow points to the leading edge apex of the eruption. This leading edge is a smooth bent curve at the beginning of the eruption as with SDO observations (Figures 1 and 2). The filament feet appearance below the filament are partly different than the ones observed by AIA because STEREO A observes the other side of the filament. As the eruption proceeds, the filament becomes bright both because of plasma heating, as described above for AIA observations, and because the plasma is observed over the limb with a weaker emitting background, so as a prominence. Next, a large quiescent prominence is present in the background at the eastern limb (Figure 5). This corresponds to an extended filament, about one solar radius long, located in between the diffuse eastern extension of the positive polarity of AR 11690 (well outside the field of view of Figure 3) and another large scale and diffuse negative polarity located further to the East. No significant consequence of the eruption of $\mathrm{Fil}_{1}$ is observed on this large quiescent filament (Figure 5).

By combining the data of AIA $304 \AA$ and STEREO A $304 \AA$ data, these stereoscopic observations allow to derive the true height of the filament. The location pointed by the arrow in Figure 5 is taken for the computation of the filament top height using the trigonometric triangulation method "ssc_measure" available in solar soft. The points selected for the height-time plot in triangulation method are along the direction of slice $S_{1}$ for AIA observations. This provides us an opportunity to compare the calculated speeds derived by the time-distance analysis and the stereoscopic analysis. For the comparison of the height derived from the triangulation method and from the AIA time-distance analysis, the common height reference is set before the eruption at the top of the filament. The calculated heights are overplotted on the time-distance plot of Figure $4 \mathrm{~b}$ with the blue ' + ' symbol. Using these points, we compute a mean speed of $\approx 180 \mathrm{~km} \mathrm{~s}^{-1}$. This derived speed from triangulation methods is about $20 \%$ faster than the speed derived from the time-distance analysis of the AIA data. This difference is due to projection effects as the velocity measured with AIA does not include the velocity component out of the plane of sky.

Finally, the filament eruption produced a CME observed by SOHO/LASCO in the north-west direction. In LASCO C2 field of view the CME appear $\approx 14: 48 \mathrm{UT}$ at a height of $4 R_{\odot}$ and it is visible in LASCO C3 field of view up to $\approx 25 R_{\odot}$ at 19:54 UT. The CME was a partial halo having a projected width of $323^{\circ}$. The measured mean speed within $\mathrm{C} 2$ and $\mathrm{C} 3$ fields of view is $\approx 790 \mathrm{~km} \mathrm{~s}^{-1}$ (see https://cdaw.gsfc.nasa.gov/CME_list), then the ejection was further accelerated compare to the above measurements in the low corona. 


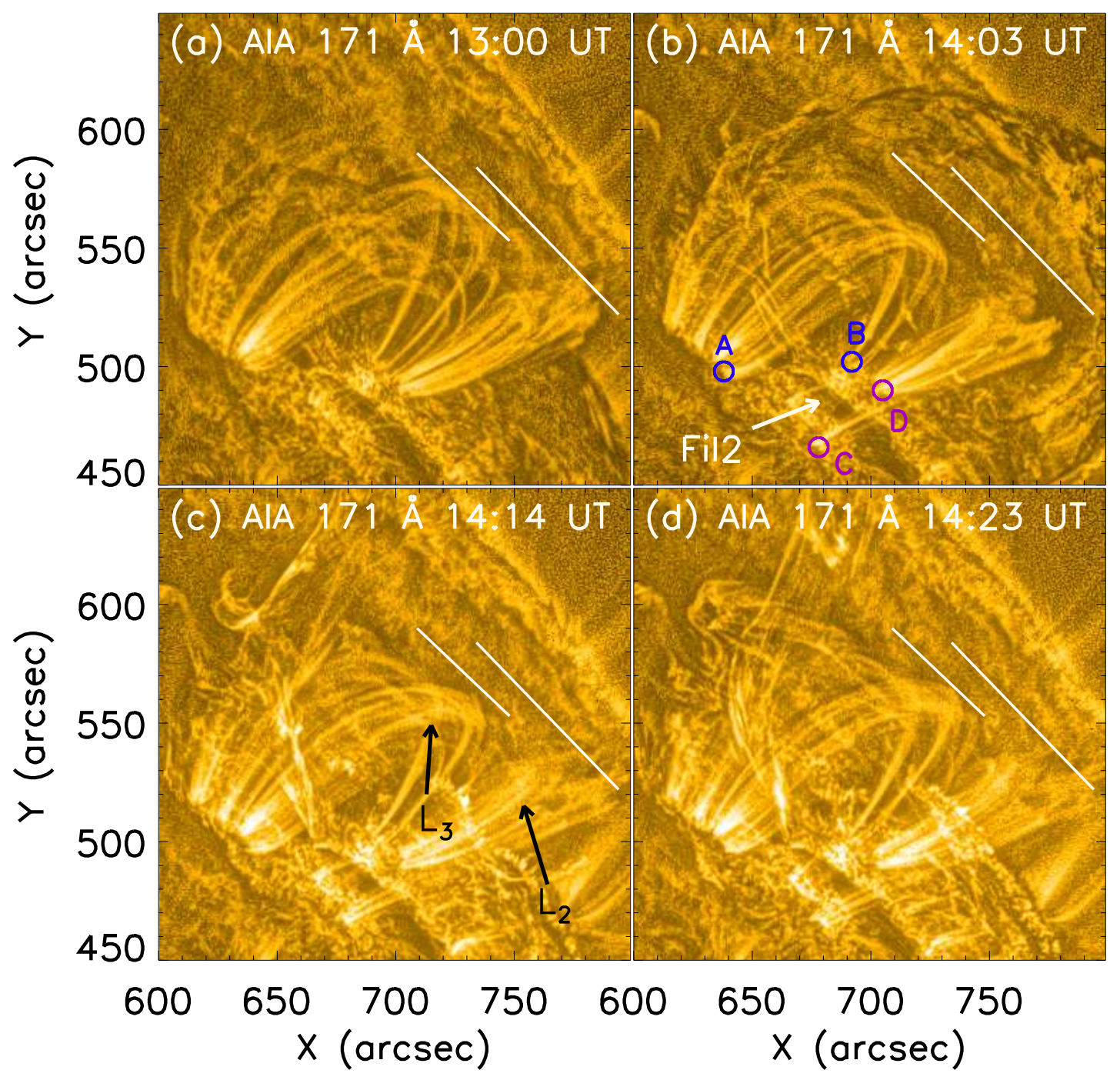

Fig. 6.- Evolution of the two loop sets located in the neighborhood of the filament Fil 1 eruption and above $\mathrm{Fil}_{2}$ (seen in the top part of panel (b)). The data are from AIA $171 \AA$ processed with the MGN technic. The white lines are at fixed positions in the solar frame to better visualize the loop evolution. After the filament eruption onset at $\approx 13: 19 \mathrm{UT}$, the loops first contract (panel b), then they expand backward towards their initial locations (panels $\mathrm{c}$ and d). The two loop systems are labeled with $\mathrm{L}_{3}$ and $\mathrm{L}_{2}$ in panel (c). The selected loop footpoints are marked with $\mathrm{A}, \mathrm{B}$, and $\mathrm{C}, \mathrm{D}$, respectively, in panel (b), and they are reported in Figure 3 f 


\subsection{Loops Contraction and Expansion}

In this section, we analyse the EUV loop contraction and expansion related to the eruption of the filament $\mathrm{Fil}_{1}$ on 2013 March 16. We will not study the shrinkage of flare loops $\mathrm{L}_{1}$ as this phenomena was already well studied (see Section 1) but rather the evolution of coronal loops which are not involved in the flare reconnection. This phenomena is observed with 171 and $193 \AA$ filters in two set of loops (Figure 6). We name these two loop systems as $\mathrm{L}_{2}$ and $\mathrm{L}_{3}$, respectively, as indicated in Figures $2 \mathrm{p}$ and 6c. Both loop systems are rooted in $P_{1}$ (positive) and $N_{1}$ (negative) polarities (Figure 3, f) of AR 11690. The location of STEREO A compare to SDO is suited for a triangulation, as done above for $\mathrm{Fil}_{1}$, however this is not possible for the loops of $\mathrm{L}_{2}$ and $\mathrm{L}_{3}$ as they are hidden behind the filament Fil $_{1}$ (Figure 5).

The eastern set, $\mathrm{L}_{3}$, is better observed with loops seen from one side, nearly face on, and they are well defined over their full length. These loops are located above the location where filament $\mathrm{Fil}_{1}$ and $\mathrm{Fil}_{2}$ are nearly joining (Figure 6b,c). They end on both sides of these filaments (Figure $3 \mathrm{~d}, \mathrm{f}$ ). The geometry of the western set $\mathrm{L}_{2}$ is more difficult to define from AIA observations since the direction of observation is nearly along the loops (side on). Moreover, the part closer to the observer is faint, and the emitted light is mixed with the one of the background coming from the stable filament $\mathrm{Fil}_{2}$ and its surrounding brightenings. Still, the bottom part of these legs of the loop system $\mathrm{L}_{2}$ can be seen on the front side of the filament Fil ${ }_{2}$. Then, the loops $\mathrm{L}_{2}$ are rooted on both sides of filament $\mathrm{Fil}_{2}$, as the loop system $\mathrm{L}_{3}$ (Figure $3 \mathrm{~d}, \mathrm{f}$ ). The corresponding co-aligned photospheric magnetograms, e.g., Figure 2 $\mathrm{d}$, on March 16 confirm that both sets of loops are rooted in the magnetic polarities surrounding the stable filament and the end of the erupting one. With a space filling coronal magnetic field (low plasma $\beta$ conditions), $\mathrm{L}_{2}$ and $\mathrm{L}_{3}$ belong to the same magnetic arcade passing over $\mathrm{Fil}_{2}$ and extending all along polarities $P_{1}$ and $N_{1}$. The heating is probably not large enough in the arcade middle to create dense enough coronal plasma, then there is a gap of coronal emission and the appearance of two separate sets of loops. We conclude that these two sets of loops belong to the magnetic arcade which is overlaying the stable filament Fil ${ }_{2}$ outlined with a blue contour in Figure $3 \mathrm{~d}$ and end above the northern end part of filament Fil $_{1}$.

The evolution of these loops during the filament eruption is shown in Figure 6 at four times with AIA $171 \AA$ filter. We have drawn two straight lines at the top of the loops at 13:00 UT (before the eruption onset). These lines are repeated in the next panels with fixed coordinates in the local reference frame, so by taking into account the solar rotation. Then, these lines allow to better visualize the loop evolution. We also refer to the attached movies at AIA 171 and $193 \AA$ wavelengths to view the full evolution of these loops. These movies show that all these loops are contracting in phase. The contraction is maximal about 43 min after the filament eruption onset (Figure 6b). Later on their motions reverse and they expand approximately back to their original positions. No further oscillation is observed. 

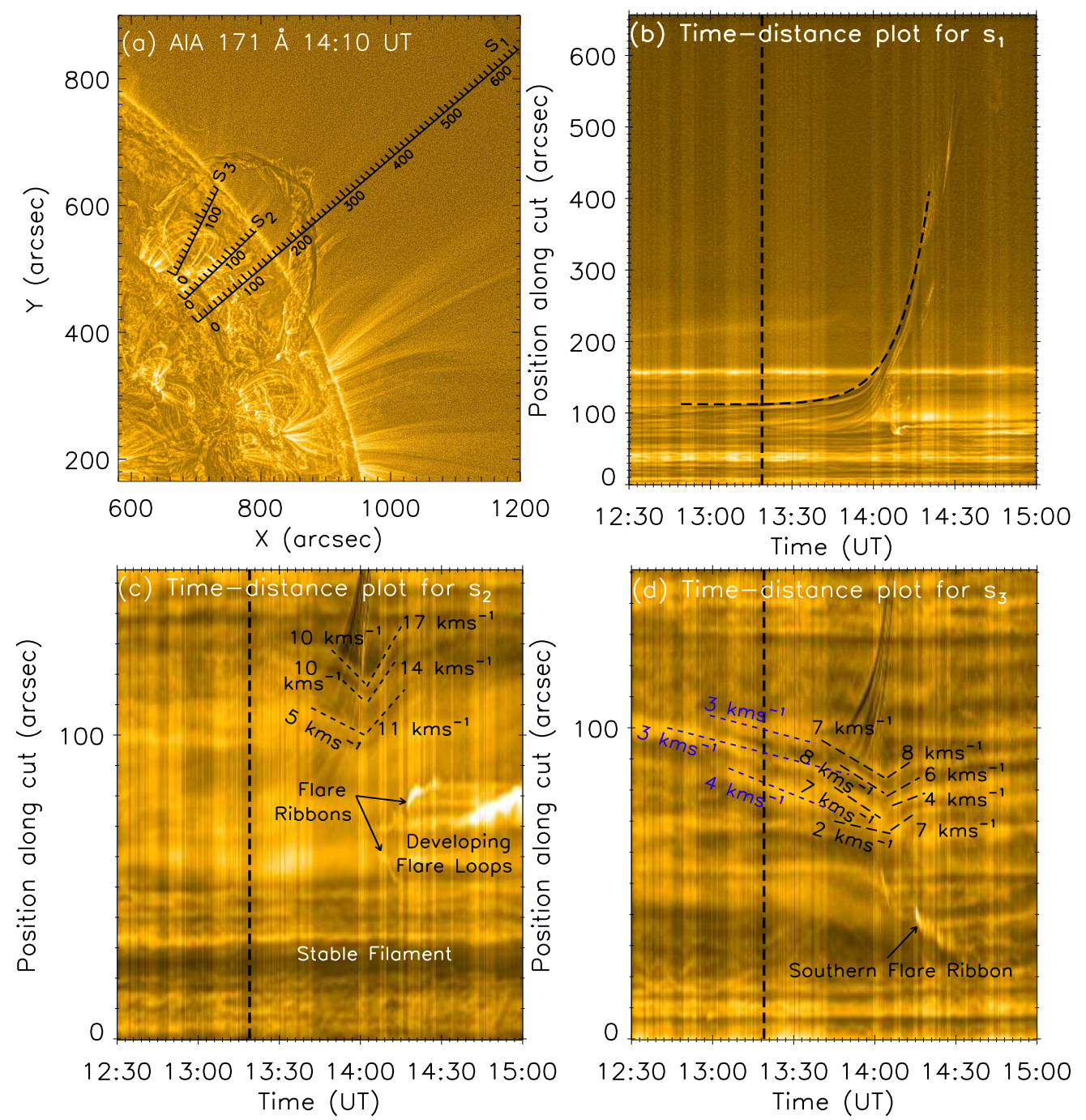

Fig. 7.- (a) AIA $171 \AA$ image processed with the MGN technic. The location of three slices are shown. Slice $S_{1}$ is the same as in Figure 4 a. It monitors the filament height versus time. Slices $S_{2}$ and $S_{3}$ are monitoring the loop contraction/expansion along the loop systems $L_{2}$ and $L_{3}$, respectively (see Figure 6). (b) Height-time plot for the filament eruption with the fit of Equation (1) to the AIA 171 data of the filament leading edge added with a dashed line. (c, d) Height-time plots along slices $S_{2}$ and $S_{3}$ showing the contraction and expansion of the EUV loops during the filament eruption. The vertical dashed line indicates the approximate onset time of the eruption. The short dashed segments outline the loop contraction/expansion of the loops. The derived mean speeds are added close to each segment. In panel (c) the trace of the flare ribbons and of the developing flare loops are indicated. In both panels $(\mathrm{c}, \mathrm{d})$ the erupting filament is seen when it emerged from the occulting coronal loops $\mathrm{L}_{2}$ and $\mathrm{L}_{3}$. 

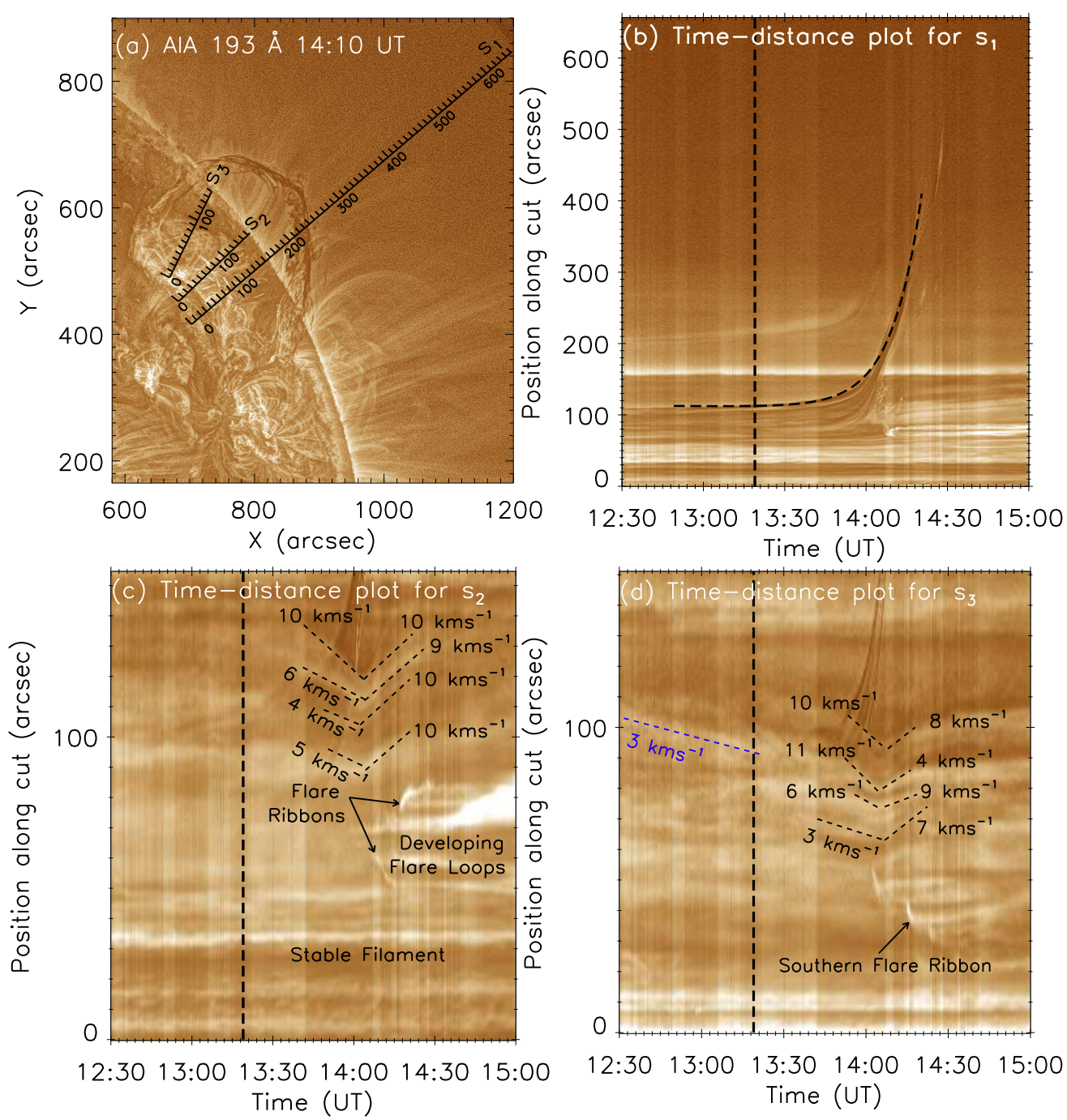

Fig. 8.- Data of AIA $193 \AA$ processed with the MGN technic and with the same format as Figure 7. (a) AIA $193 \AA$ image with the same slices $\left(S_{1}, S_{2}\right.$ and $\left.S_{3}\right)$ than in Figure 7 a. (b) Heighttime plot for the filament eruption with the fit of Equation (1) to the AIA 193 data of the filament leading edge added with a dashed line. The height has a constant projection factor on the plane of sky. (c, d) Height-time plots showing the contraction and expansion of the EUV loops after the filament eruption along slices $S_{2}$ and $S_{3}$. The vertical dashed line indicate the onset time of eruption. The typical contraction/expansion speeds of the loops are added, as well as the flare traces. 
For a quantitative analysis of these two sets of loops, we show the results obtained along two slices selected to cross the loop tops nearly orthogonally, so along the loop motion. We name these slices $S_{2}$ and $S_{3}$. They cross the loop sets $L_{2}$ and $L_{3}$, respectively. The results of these space-time analysis is presented in Figures 7 and 8 for the AIA 171 and $193 \AA$ data, respectively. We corrected the height projection from the solar rotation. The results with slice $S_{1}$, defined in Figure $4 \mathrm{a}$, are also included in the panels (b). They both confirm the upward motion and velocities of the filament $\mathrm{Fil}_{1}$ obtained with the $304 \AA$ filter. In contrast, the stable filament $\mathrm{Fil}_{2}$ is present at a nearly constant position in the lower part of panels (c) of Figures 7 and 8 . The upward little drift of $\mathrm{Fil}_{2}$ is not due to the solar rotation as it is removed. We interpret this drift as follows. This stable filament is in a decaying AR, and cancelation occurs at the PIL, further building up the FR configuration. So the FR is slowly moving up towards a nearby equilibrium. Such slow evolution has been analyzed before for several observed events (e.g., Byrne et al. 2014; Gosain et al. 2016; Chandra et al.2017).

The loops $\mathrm{L}_{2}$ crossed by slice $\mathrm{S}_{2}$ are initially stable up to $\approx 13: 40$ UT when they start contracting (Figure $7 \mathrm{c}$ ). At that time, the apex of filament Fil ${ }_{1}$ was already moving upward at a velocity $\approx 6 \mathrm{~km} \mathrm{~s}^{-1}$ (Figure 7b). Across slice $\mathrm{S}_{2}$, the erupting filament $\mathrm{Fil}_{1}$ is partly masked by the foreground set of loops $\mathrm{L}_{2}$ present along the line of sight (Figures 6, 7f). Fil 1 is observed in absorption before and after the crossing of the contracting loops. The contraction of all the loops starts almost simultaneously $\approx 21 \mathrm{~min}$ after the filament eruption onset. This is in the range 4 to $40 \mathrm{~min}$ of the time difference between the onsets of filament eruption and loop contraction reported before (Shen et al. 2014; Dudík et al. 2016, 2017; Wang et al. 2018; Devi et al. 2021). The speed of the contraction varies from 5 to $10 \mathrm{~km} \mathrm{~s}^{-1}$. This contraction continues for a duration of $\approx 22 \mathrm{~min}$. At $\approx 14: 02 \mathrm{UT}$, these loops change suddenly from contraction to expansion without any significant phase difference between them. The speed of the expansion is in the range 11 to $17 \mathrm{~km} \mathrm{~s}^{-1}$. This expansion speed is higher by a factor 2 than the contraction speed. The expansion continues at least for $15 \mathrm{~min}$, and afterwards these loops $\mathrm{L}_{2}$ are difficult to detect with the $171 \AA$ filter.

A similar loop evolution is present with AIA $193 \AA$ (Figure 8). The speeds of the contracting and expanding loops are comparable to the one observed in AIA $171 \AA$. The small difference in velocities could have several origins: the difference in the emitting plasma, the diffuse appearance of the loops, the short and partly different duration of the contraction and expansion phases (as could be observed when enough emitting plasma was present). Finally, one more loop, with similar time evolution than others, is detected in $193 \AA$ (the lower one in Figure $8 \mathrm{c}$ ).

The slice $S_{3}$ analyses a different set of loops, $L_{3}$, which are located closer to the northern leg of the erupting filament Fil $1_{1}$ (Figure 7a). This loop system has a similar behavior as the loop system $\mathrm{L}_{2}$ except that it shows also a contraction before the filament eruption onset. We refer this contraction as 'earlier loop contraction'. This earlier loop contraction can be clearly seen in the 
panels (d) of Figures 7 and 8 respectively (see also the accompanying movie). This contraction starts between $\approx 12: 30$ and 13:00 UT and the computed speed for the earlier contraction is about 3-4 $\mathrm{km} \mathrm{s}^{-1}$. Next, about $21 \mathrm{~min}$ after the eruption onset, the loops in $\mathrm{S}_{3}$ contract as the ones of slice $S_{2}$. The contraction speed in the case of $171 \AA$ varies from 2 to $8 \mathrm{~km} \mathrm{~s}^{-1}$ which is comparable to the speeds deduced from the $193 \AA$ filter (in the range 3 to $10 \mathrm{~km} \mathrm{~s}^{-1}$ ). The contraction time for both wavelengths is about $22 \mathrm{~min}$. As the loops of $\mathrm{S}_{2}$, the contraction changes rapidly to an expansion at $\approx 14: 02 \mathrm{UT}$. The expansion speed varies from 4 to $8 \mathrm{~km} \mathrm{~s}^{-1}$ in case of $171 \AA$ and from 4 to $9 \mathrm{~km} \mathrm{~s}^{-1}$ in the case of $193 \AA$ so comparable to the contraction speed. For both contraction and expansion, there is a global tendency of an increasing velocity with height, a tendency which is also present for slice $S_{2}$. Next, at the difference with loops $\mathrm{L}_{2}$, loops $\mathrm{L}_{3}$ could be followed much longer in time. About 20 min after motion reversal, this expansion slows down and it is over by $\approx$ 14:27 UT (panels (d) of Figures 7) and 8). No significant evolution is present later on.

The slice $\mathrm{S}_{2}$ shows also the consequences of the flare reconnection (panels (c) of Figures 7 and 8 ). The flare ribbons start to significantly brighten and to separate from each others in slice $S_{2}$ at $\approx 14: 18 \mathrm{UT}$, while they are detected starting earlier, at $\approx 13: 59 \mathrm{UT}$, close to slice $\mathrm{S}_{3}$ which is coherent with an eruption starting at the end of Fil $1_{1}$. The flare loops are observed later on, after $\approx$ 14:34 UT in $\mathrm{S}_{2}$. The drift of position observed in panels (c) of Figures 7 and 8 is interpreted as the formation of higher flare loops as magnetic reconnection proceeds. In slice $S_{3}$, after 14:00 UT and for an abscissa $<50$ "there is a brightening shift to lower $\mathrm{S}_{3}$ abscissa. We have examined this shift in detail by analyzing the movies and corresponding images. This shift corresponds to the motion of the southern flare ribbon.

\section{Physical Interpretation}

\subsection{Erupting Magnetic Field Configuration}

The evolution of the radial magnetic field component is typical on an AR in decay (Figure 3 and associated movie). Magnetic flux is progressively dispersed by super-granules convective cells. This implies that both magnetic polarities of AR 11690 are growing in extension. This induced magnetic field cancelations around the photospheric level along the internal PIL of the AR. This evolution is generically expected to build a FR (van Ballegooijen \& Martens 1989; Aulanier et al. 2010; Green et al. 2011) where dense plasma could be caught (e.g., Aulanier \& Démoulin 1998), forming filament $\mathrm{Fil}_{2}$.

Photospheric magnetic cancelation is also triggered by the field dispersion at the AR boundary, especially at the PIL between polarities $\mathrm{N}_{1}$ and $\mathrm{P}_{0}$. Our observations of cancellation of mag-

netic flux at the photospheric level below the filaments are consistent with the study of the filament 
formation of Wang \& Muglach (2007). Two of their studied filaments were formed at the periphery of ARs, like Fil $1_{1}$, while the third one was formed inside a decaying AR like Fil ${ }_{2}$. They concluded that the filaments are formed due to the same process of magnetic flux cancellation at photospheric inversion line. Here, the studied filaments, $\mathrm{Fil}_{1}$ and $\mathrm{Fil}_{2}$, are around the same magnetic polarity $\mathrm{N}_{1}$. Before the eruption it is difficult to set the limit between Fil ${ }_{1}$ and $\mathrm{Fil}_{2}$ but eruption of only Fil favours that they have two separated magnetic configurations.

The main add to the above magnetic diffusion scenario is the emergence of a magnetic bipole in the leading negative polarity of AR 11690 (Figure 3b-e and associated movie). This bipole has almost the reverse orientation than the main bipole forming AR $11690\left(\mathrm{P}_{1}, \mathrm{~N}_{1}\right)$. The emerging positive polarity mainly cancels with the outer (westward) part of polarity $\mathrm{N}_{1}$. This reconnection transfers the negative footpoint from $\mathrm{N}_{1}$ to the emerging negative polarity. The coronal implication of this reconnection depends on the initial coronal connectivity of $\mathrm{N}_{1}$. The part closer to the internal PIL of AR 11690 is expected to connect to $P_{1}$. In this case the reconnection brings the negative footpoint of the reconnected fields connecting $\mathrm{P}_{1}$ closer to the internal PIL. This decreases the curvature radius of field lines, then strengthen the stabilizing magnetic tension force of the magnetic configuration supporting filament Fil 2 . However, the external part of $\mathrm{N}_{1}$ is expected to connect $\mathrm{P}_{0}$ (e.g., as it does with a potential field). In this case the reconnection brings the negative footpoint of the reconnected fields connecting $\mathrm{P}_{0}$ away from the external PIL. This weaken the stabilizing tension force of the filament $\mathrm{Fil}_{1}$, then it is an ingredient to bring the magnetic configuration of $\mathrm{Fil}_{1}$ to eruption. Still, this reconnection was not sufficient since the positive polarity of the emergence canceled and disappeared on day before $\mathrm{Fil}_{1}$ eruption. These results are in agreement with the study of Chen \& Shibata (2000).

The 3D standard model of eruptive flares is typically developed in a bipolar field modeling an AR with the build up, then eruption, of a FR formed above the internal PIL (e.g., Aulanier et al. 2010; Janvier et al. 2015). In the case of an external PIL, located at periphery an AR, the same model could be qualitatively be applied if a first coronal reconnection forms a sheared arcade over this PIL, as shown by Török et al. (2018). The following evolution is mainly driven by the diffusion of magnetic polarities, as for the case of an internal PIL. The main difference at this stage is an expected slower process as the spatial region involved around the external PIL is large while convective cells have typically the same speed.

When the magnetic configuration reaches an instability (as evidenced by the exponential growth of the filament height), the filament trace an erupting FR structure well observed both with SDO/AIA and STEREO A/EUVI $304 \AA$ filters (Figures 4 and 5 and related movies). Reconnection behind the erupting FR leads to the formation of a flare loop arcade ending in two J-shaped flare ribbons observed in EUV wavelengths. They separate as a function of time as excepted in the standard 3D eruption model. Then, we conclude that, while located at the periphery of an AR, the 
eruption of $\mathrm{Fil}_{1}$ has all the characteristics expected with the 3D standard model of eruptive flares build for eruptions located in the core of ARs. The main differences are slower processes both for the formation and the ejection of the FR (due to a weaker magnetic field, so weaker forces).

\subsection{Contracting and Expanding Loops}

The loop system $\mathrm{L}_{3}$ (panel (d) of Figures 7)and 8) shows the earlier contraction as mentioned in Section 2.4. The data presented here provide no clue about this contraction. We observe small brightenings at the base of loop system $\mathrm{L}_{3}$ before the eruption and a short description of our analysis is presented in appendix A since this could be interesting for further studies on the subject. In present observations the sets of contracting loops, $\mathrm{L}_{2}$ and $\mathrm{L}_{3}$, mostly recover their original heights they had when the fast contraction started (Figure 6, panels (c) and (d) of Figures 7 and 8). This evolution is different than for loops located in the elbow of AR sigmoids as, the expansion following contraction is frequently not able to recover the pre-eruption location (e.g., Liu et al. 2012; Simões et al. 2013; Shen et al. 2014; Wang et al. 2018) while in some cases it does (e.g., Dudík et al. 2017). This behaviour is expected for loops at a remote filament channel where no energy release happens. A possibility is that the eruption generates a coronal wave which first pushes the loops $\mathrm{L}_{2}$ and $\mathrm{L}_{3}$ downward, then the loops recover their initial positions after the coronal wave passage. Indeed, there have been previously studied cases where loops oscillate during the crossing of a coronal wave (e.g., Ballai 2007; Guo et al. 2015; Fulara et al. 2019). In these studies, the oscillations have at least one cycle. In the present case, a weak coronal wave is associated with the eruption as visible at http://suntoday.lmsal.com/sdomedia/SunInTime/2013/ 03/16/AIAtriratio-211-193-171-2013-03-16T1200.mov.mp4. Due to the weak nature of coronal wave, it is very difficult to estimate if it interacted with the loops and at which time. The main observational constraint is that the loops have only half period oscillation with a triangular shaped amplitude. This is far from the behavior expected from an unforced oscillator, as summarized e.g., in Figures 2 and 4 of Russell et al. (2015). The observed triangular shape in Figures 7 and 8 is also well different from oscillations observed in other events (e.g., Gosain 2012; Liu et al. 2012, Simões et al. 2013). Then, an excitation of the loops by a coronal wave is doubtful.

An additional possibility for the origin of the loop contraction and expansion is a perturbation by the lateral expansion of the erupting flux of the filament. It is known that some eruptions show a so-called overexpansion, a stronger growth of the minor radius (thickness) of the erupting flux compared to the major radius (height) (Patsourakos et al. 2010ab; Veronig et al. 2018). In the case of Veronig et al. (2018), the lateral overexpansion of the CME bubble first pushes neighboring loops to the side, which leads to very clear oscillations. Subsequently, the bubble acquires a

mushroom-like shape, which presses some loops to the north of the eruption downward, followed 
by a recovery of the loops to the original height. Such behavior was so far seen preferentially in fast or impulsive eruptions. However, a similar dynamics in slower eruptions, like the case here, might nevertheless be possible. Since the perturbation is temporary, a return of the loops to their original position would result naturally.

This event shows, as expected by filament models, that the erupting flux is much thicker and extends much higher than the filament in the hotter channel $211 \AA$. A diffuse front is seen to rise synchronously with the filament at nearly twice the height (panel $(j)$ of Figure 1). The synchronous motion suggests that the diffuse front is part of the erupting flux, probably at its top edge. This implies that most of the erupting flux is already higher than the loops $\mathrm{L}_{2}$ and $\mathrm{L}_{3}$ when their contraction begins. The lateral expansion of the flux at this stage should perturb the flux above these loops. When, as the overlying flux is pushed sideward by the CME bubble, the loops may experience a sideward and downward push. Another possibility of this moving diffuse front at the top of the erupting FR can be formed by the erupting FR, which collects, compress and heats surrounding coronal plasma (and magnetic field too) in front of the FR. So it could not be a part of the erupting flux but coronal plasma collected on the way. It is likely the beginning of a sheath formation, a structure well observed in-situ in front of magnetic clouds and more generally ejecta far away from the Sun.

We next analyse how other proposed mechanisms may explain these observations. The implosion conjecture is difficult to test since it was so far not quantitatively formalised and moreover its physical base changed (e.g. from a driver to a consequence of the eruption, see Section 1 ). Indeed, this conjecture would have to explain how the loop contraction could occur above the stable filament $\mathrm{Fil}_{2}$ located inside an AR, while the eruption of another filament Fil ${ }_{1}$ occurs at the leading border of the same AR. This requires an analysis, with a numerical simulation, which is outside the scope of present study. Still, the observations show that the northern end of filament Fil $_{1}$ enters slightly within the AR, and reach an end location below $\mathrm{L}_{3}$ loops. Then, the eruption of $\mathrm{Fil}_{1}$ is clearly involved in this loop contraction while we cannot provide elements in support of the implosion conjecture. Even more, the evolution of $\mathrm{L}_{2}$ loops above the stable filament $\mathrm{Fil}_{2}$ and the return of the loops to their initial position are not within the framework of the implosion conjecture.

The loop contraction model of Zuccarello et al. (2017) explained loop contraction by the development of magnetohydrodynamic vortexes which develop on the sides of an erupting FR. The loop contraction, then expansion occurs in the field lines overlying the footpoints of the FR. The numerical simulations study the instability of a FR located in a bipolar field simulating a simple and isolated AR. This configuration is closer to the magnetic configuration of and above the stable filament $\mathrm{Fil}_{2}$, than of the one of the erupting filament Fil ${ }_{1}$. Still, following the strongly bended PIL, the contraction/expansion occur on the loops which are next to the northern footpoint of the 
erupting FR. Then, the main difference with the simulations of Zuccarello et al. (2017) is that the PIL is so bent that the contracting loops appear on one lateral side, rather than in the continuation, of the erupting filament. Still, an MHD simulation with the observed multipolar configuration (Figure 3) is needed to test the possibility that magnetohydrodynamic vortexes develop and imply the loop contraction/expansion as found before with a simple bipolar magnetic configuration.

\section{Conclusion}

We analyze a filament eruption of 2013 March 16 located close to the west limb. The filament, $\mathrm{Fil}_{1}$, was located in between the leading polarity of a decaying active region and a westward remnant dispersed polarity of opposite sign. In this weak magnetic field environment no GOES flare was reported associated to this eruption. Still, we report an arcade of flare loops and two separating J-shaped flare ribbons on the opposite sides of the PIL of the erupting filament. Later on, the eruption leads to a CME with moderate speed (about $800 \mathrm{~km} \mathrm{~s}^{-1}$ ). All these observational characteristics fit well within the standard 3D model of solar eruptions (Section 1).

The dynamics of the erupting filament is well fitted with a model adding a linear and an exponential increases of height with time. This quantifies two erupting phases: a slow rise and an acceleration phases which characterize two different physical mechanisms. A slow rise is indeed expected to occur as a consequence of the observed cancelation of the photospheric magnetic field at the PIL. This process is expected to build a FR which is progressively rising in height towards a new equilibrium. Recurrent brightenings and restructuration are observed at the northern footpoint of the filament during this phase. Next, the exponential growth of height during the acceleration phase characterizes an instability, which is likely the torus instability since no significant writhing of the erupting filament is observed (as would be present for a kink instability). The onset of the instability is estimated with the time when the exponential growth of the upward velocity becomes significant on top of the previous nearly constant velocity. Another remarkable aspect of the filament eruption was the observations of the drift of southern foot-points of the erupting FR, which could be explained by the interchange reconnection of the erupting configuration with the confront magnetic loops of a neighbouring active region. Moreover, we also observe the split of the eruptive FR above the southern foot-points with plasma tracing the magnetic connections to two separated magnetic polarities.

Another filament, $\mathrm{Fil}_{2}$, is present along the internal PIL of the AR. The northern ends of the filaments are close by, so that both filaments encircle almost the leading polarity of the active region. It is then remarkable that $\mathrm{Fil}_{2}$ stays undisturbed while Fil 1 is erupting. About 21 min after $\mathrm{Fil}_{1}$ eruption onset, the loops $\mathrm{L}_{3}$ suddenly accelerate their contraction speed by a factor 2 to 3 . At the same time the initially stable southern loops $\mathrm{L}_{2}$, located above Fil ${ }_{2}$, contract in a similar 
way. Then, the full coronal loop system contract for a period of $22 \mathrm{~min}$. Later on, the loop motion reverses suddenly, then they expand for a period of 20-25 min. The amplitude of the contraction is in between 10 and $20^{\prime \prime}$. Then, this evolution is well resolved both spatially and temporally by SDO/AIA observations. Both the contraction and expansion were observed in phase in the full loop arcade on a significant range of projected heights range (about 70 "). Afterwards, these loops set into a stable configuration close to their original positions at the start of the fast contraction phase. The return of the loops to the original position is naturally expected because they pass over the end point of $\mathrm{Fil}_{1}$ and over $\mathrm{Fil}_{2}$, where no or only minimal energy release is expected to occur and the post-eruption equilibrium should be close to the pre-eruption equilibrium.

The characteristics of the loop contraction/expansion in this eruption contrast with previous studies. First, the loop arcade is located above a stable filament and extends up to one end of another erupting filament. Second, the stable filament is located mostly parallel to the erupting one. Third, the stable and erupting filaments are on the same PIL encircling the leading PIL of an active region in decay. Finally, the loop evolution starts with a contraction followed by an expansion recovery phase. This half period oscillation with a triangular shape is an original characteristic of present event. All these characteristics make this studied event challenging for the models of loop contraction since such magnetic configuration was not observed or modelled before. In particular, this case is different than the one simulated by Zuccarello et al. (2017) where the eruption occurs in the core of the simulated active region, while in present observations the eruption occur at the leading border of the active region. In summary, numerical simulations are needed with a broader variety of magnetic configuration in order to understand the results of present observations. In particular, it is worth studying how strongly the results are depending on the bending of PIL, so on the fully $3 \mathrm{D}$ aspects of the involved magnetic configuration. Such studies can also allow to separate better the characteristics of the different models proposed so far to interpret the contraction and expansion of coronal loops.

\section{Acknowledgments}

We recognize the collaborative and open nature of knowledge creation and dissemination, under the control of the academic community as expressed by Camille Noûs at http://www . cogitamus.fr/indexen.html. The authors thank the open data policy of the SDO and STEREO teams. RC acknowledges the support from Bulgarian Science Fund under Indo-Bulgarian bilateral project, DST/INT/BLR/P-11/2019. P.D. is supported by CSIR, New Delhi. We thank the reviewers for their constructive comments and suggestions. 


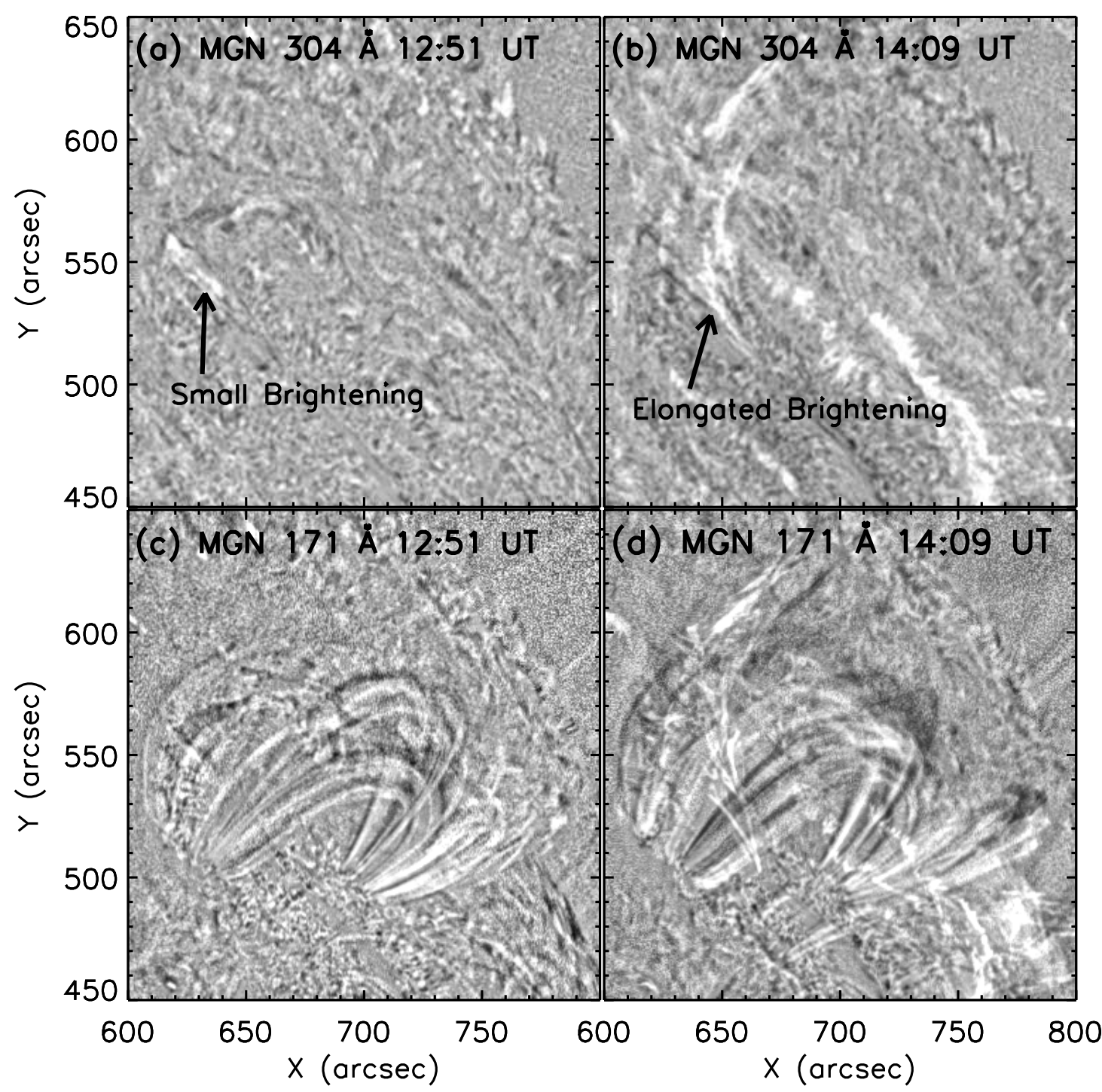

Fig. 1.- MGN base difference images of AIA 304 and $171 \AA$ at 12:51 and 14:09 UT. The base time for these images is 12:30 UT. The small brightening before the eruption and the elongated brightening during the eruption are shown in panels $(\mathrm{a}, \mathrm{c})$ and $(\mathrm{b}, \mathrm{d})$, respectively. These brightenings are located at the end of filament $\mathrm{Fil}_{1}$, close and partly below the $\mathrm{L}_{3}$ loop system (bottom panels). The associated movie is available in the Electronic Supplementary Materials. The animation starts at 12:30 UT and end at 15:00 UT. The realtime duration of the animation is 25 seconds.

\section{A. Appendix: Earlier Loop Contraction}

In order to understand the earlier contraction of loop system $\mathrm{L}_{3}$ (panels (d) of Figures 7 and 8), we studied based difference movies of AIA 304 and $171 \AA$ without and with MGN technique 
applied before subtracting the image at 12:30 UT. A main brightening episode is starting at 12:42 UT and is illustrated in the left panels of Figure 1 in the middle of its duration. This local event is associated with local plasma motions indicating a local restructuration of the magnetic field of the northern end of filament Fil 1 . A second brighter event started at 13:30 UT in a close by location, while more extended both north, along $\mathrm{Fil}_{1}$, and southward inside the AR (Figure 1, right panels). It shows that, at the beginning of eruption, Fil $_{1}$ was entering slightly inside the AR (as drawn in Figure $3 \mathrm{~d}$ ). This event is the activation and reconfiguration of Fil $1_{1}$ northern end. The start of this event is later by at least 11 minutes than the eruption onset define in Figure $4 \mathrm{p}$ (Section 2.3). These local events are best seen in $304 \AA$, while they are also present in $171 \AA$ which allows to locate them well with respect to the loops $\mathrm{L}_{3}$ (Figure 1, bottom panels). We conclude that these brightenings are the only indications present in the data of magnetic reconfiguration occurring in the vicinity of loops $\mathrm{L}_{3}$ during the linear and and early exponential phases of the filament rise. Then, the data provide no clue about the earlier contraction of loop system $\mathrm{L}_{3}$. This earlier contraction is possibly associated to the upward motion of the magnetic configuration of Fil $1_{1}$ in the linear phase. In this case, it has the same physical origin as the contraction present later on in the exponential phase (panels (c) and (d) of Figures 7]and 8).

\section{REFERENCES}

Amari, T., Aly, J. J., Mikic, Z., \& Linker, J. 2010, ApJ, 717, L26, doi: $10.1088 / 2041-8205 /$ 717/1/L26

Antiochos, S. K., Dahlburg, R. B., \& Klimchuk, J. A. 1994, ApJ, 420, L41, doi: $10.1086 /$ 187158

Antiochos, S. K., DeVore, C. R., \& Klimchuk, J. A. 1999, ApJ, 510, 485, doi: $10.1086 / 306563$

Aulanier, G., \& Démoulin, P. 1998, A\&A, 329, 1125

Aulanier, G., Démoulin, P., Mein, N., et al. 1999, A\&A, 342, 867

Aulanier, G., \& Schmieder, B. 2002, A\&A, 386, 1106, doi:10.1051/0004-6361:20020179

Aulanier, G., Török, T., Démoulin, P., \& DeLuca, E. E. 2010, ApJ, 708, 314, doi: $10.1088 /$ $0004-637 \mathrm{X} / 708 / 1 / 314$

Ballai, I. 2007, Sol. Phys., 246, 177, doi: 10.1007/s11207-007-0415-3

Ballai, I., Douglas, M., \& Marcu, A. 2008, A\&A, 488, 1125, doi: 10.1051/0004-6361: 200809833 
Byrne, J. P., Morgan, H., Seaton, D. B., Bain, H. M., \& Habbal, S. R. 2014, Sol. Phys., 289, 4545, doi: $10.1007 / \mathrm{s} 11207-014-0585-8$

Carmichael, H. 1964, NASA Special Publication, 50, 451

Chandra, R., Filippov, B., Joshi, R., \& Schmieder, B. 2017, Sol. Phys., 292, 81, doi: $10.1007 /$ S11207-017-1104-5

Chandra, R., Pariat, E., Schmieder, B., Mandrini, C. H., \& Uddin, W. 2010, Sol. Phys., 261, 127, doi: $10.1007 /$ s11207-009-9470-2

Chandra, R., Schmieder, B., Aulanier, G., \& Malherbe, J. M. 2009, Sol. Phys., 258, 53, doi: 10 . 1007/s11207-009-9392-z

Chen, P. F., \& Shibata, K. 2000, ApJ, 545, 524, doi: $10.1086 / 317803$

Cheng, X., Zhang, J., Kliem, B., et al. 2020, ApJ, 894, 85, doi: 10.3847 /1538-4357/ab886a

Démoulin, P., Priest, E. R., \& Lonie, D. P. 1996, J. Geophys. Res., 101, 7631, doi: $10.1029 /$ 95JA03558

Devi, P., Démoulin, P., Chandra, R., et al. 2021, A\&A, 647, A85, doi: $10.1051 / 0004-6361 /$ 202040042

Dudík, J., Lörinčík, J., Aulanier, G., Zemanová, A., \& Schmieder, B. 2019, ApJ, 887, 71, doi: 10 . $3847 / 1538-4357 / a b 4 \pm 86$

Dudík, J., Zuccarello, F. P., Aulanier, G., Schmieder, B., \& Démoulin, P. 2017, ApJ, 844, 54, doi: $10.3847 / 1538-4357 /$ aa 7 aab

Dudík, J., Polito, V., Janvier, M., et al. 2016, ApJ, 823, 41, doi: $10.3847 / 0004-637$ X/823/ $1 / 41$

Forbes, T. G., \& Acton, L. W. 1996, ApJ, 459, 330, doi: $10.1086 / 176896$

Fulara, A., Chandra, R., Chen, P. F., et al. 2019, Sol. Phys., 294, 56, doi: $10.1007 /$ s11207-019-1445-3

Georgoulis, M. K., Nindos, A., \& Zhang, H. 2019, Philosophical Transactions of the Royal Society of London Series A, 377, 20180094, doi: $10.1098 /$ rsta.2018.0094

Gibson, S. E., \& Fan, Y. 2006, ApJ, 637, L65, doi: $10.1086 / 500452$

Gosain, S. 2012, ApJ, 749, 85, doi: $10.1088 / 0004-637 X / 749 / 1 / 85$ 
Gosain, S., Filippov, B., Ajor Maurya, R., \& Chandra, R. 2016, ApJ, 821, 85, doi: $10.3847 /$ $0004-637 \mathrm{X} / 821 / 2 / 85$

Green, L. M., Kliem, B., \& Wallace, A. J. 2011, A\&A, 526, A2, doi: $10.1051 / 0004-6361 /$ 201015146

Green, L. M., Török, T., Vršnak, B., Manchester, W., \& Veronig, A. 2018, Space Sci. Rev., 214, 46, doi: $10.1007 /$ s11214-017-0462-5

Guo, Y., Erdélyi, R., Srivastava, A. K., et al. 2015, ApJ, 799, 151, doi: $10.1088 / 0004-637 X /$ $799 / 2 / 151$

Hirayama, T. 1974, Sol. Phys., 34, 323, doi: $10.1007 /$ BF 00153671

Hori, K. 2000, ApJ, 543, 1011, doi: $10.1086 / 317162$

Howard, R. A., Moses, J. D., Vourlidas, A., et al. 2008, Space Sci. Rev., 136, 67, doi: $10.1007 /$ S11214-008-9341-4

Hudson, H. S. 2000, ApJ, 531, L75, doi: $10.1086 / 312516$

Janvier, M., Aulanier, G., \& Démoulin, P. 2015, Sol. Phys., 290, 3425, doi: $10.1007 /$ s11207-015-0710-3

Joshi, B., Veronig, A., Cho, K. S., et al. 2009, ApJ, 706, 1438, doi: 10.1088/0004-637X/ $706 / 2 / 1438$

Kay, C., Opher, M., \& Evans, R. M. 2015, ApJ, 805, 168, doi: 10.1088/0004-637X/805/ $2 / 168$

Kippenhahn, R., \& Schlüter, A. 1957, Zeitschrift fur Astrophysik, 43, 36

Kliem, B., \& Török, T. 2006, Phys. Rev. Lett., 96, 255002, doi: 10.1103 / PhysRevLett . 96. 255002

Kliem, B., Török, T., Titov, V. S., et al. 2014, ApJ, 792, 107, doi: $10.1088 / 0004-637$ X/792/ $2 / 107$

Kopp, R. A., \& Pneuman, G. W. 1976, Sol. Phys., 50, 85, doi: 10.1007 /BF 00206193

Labrosse, N., Heinzel, P., Vial, J. C., et al. 2010, Space Sci. Rev., 151, 243, doi: $10.1007 /$ s11214-010-9630-6

Lemen, J. R., Title, A. M., Akin, D. J., et al. 2012, Sol. Phys., 275, 17, doi: $10.1007 /$ S11207-011-9776-8 
Li, Y. P., \& Gan, W. Q. 2006, ApJ, 644, L97, doi: $10.1086 / 505576$

Lin, J., \& Forbes, T. G. 2000, J. Geophys. Res., 105, 2375, doi: 10 . $1029 / 1999$ JA 900477

Liu, R., Liu, C., Török, T., Wang, Y., \& Wang, H. 2012, ApJ, 757, 150, doi: $10.1088 /$ $0004-637 \mathrm{X} / 757 / 2 / 150$

Lörinčík, J., Dudík, J., \& Aulanier, G. 2019, ApJ, 885, 83, doi: 10.3847/1538-4357/ ab4519

Mackay, D. H., Karpen, J. T., Ballester, J. L., Schmieder, B., \& Aulanier, G. 2010, Space Sci. Rev., 151, 333, doi: $10.1007 /$ s11214-010-9628-0

Moore, R. L., \& Sterling, A. C. 2006, Washington DC American Geophysical Union Geophysical Monograph Series, 165, 43, doi: $10.1029 / 165$ GM0 7

Morgan, H., \& Druckmüller, M. 2014, Sol. Phys., 289, 2945, doi: $10.1007 /$ s11207-014-0523-9

Panasenco, O., Martin, S. F., Velli, M., \& Vourlidas, A. 2013, Sol. Phys., 287, 391, doi: 10 . 1007/s11207-012-0194-3

Parenti, S. 2014, Living Rev. in Sol. Phys., 11, 1, doi: 10.12942 / 1rsp-2014-1

Patsourakos, S., Vourlidas, A., \& Kliem, B. 2010a, A\&A, 522, A100, doi: 10.1051/ $0004-6361 / 200913599$

Patsourakos, S., Vourlidas, A., \& Stenborg, G. 2010b, ApJ, 724, L188, doi: $10.1088 /$ 2041-8205/724/2/L188

Patsourakos, S., Vourlidas, A., Török, T., et al. 2020, Space Sci. Rev., 216, 131, doi: $10.1007 /$ s11214-020-00757-9

Pesnell, W. D., Thompson, B. J., \& Chamberlin, P. C. 2012, Sol. Phys., 275, 3, doi: $10.1007 /$ s11207-011-9841-3

Priest, E. R., Hood, A. W., \& Anzer, U. 1989, ApJ, 344, 1010, doi: $10.1086 / 167868$

Russell, A. J. B., Simões, P. J. A., \& Fletcher, L. 2015, A\&A, 581, A8, doi: $10.1051 /$ $0004-6361 / 201525746$

Schmieder, B., Démoulin, P., \& Aulanier, G. 2013, Adv. Space Res. , 51, 1967, doi: $10.1016 /$ j.asr.2012.12.026 
Schou, J., Scherrer, P. H., Bush, R. I., et al. 2012, Sol. Phys., 275, 229, doi: $10.1007 /$ s11207-011-9842-2

Shen, J., Zhou, T., Ji, H., et al. 2014, ApJ, 791, 83, doi: $10.1088 / 0004-637 X / 791 / 2 / 83$

Simões, P. J. A., Fletcher, L., Hudson, H. S., \& Russell, A. J. B. 2013, ApJ, 777, 152, doi: 10 . $1088 / 0004-637 \mathrm{X} / 777 / 2 / 152$

Sturrock, P. A. 1966, Nature, 211, 695, doi: $10.1038 / 211695$ a 0

Sui, L., Holman, G. D., \& Dennis, B. R. 2004, ApJ, 612, 546, doi: 10 . $1086 / 422515$

Sun, X., Hoeksema, J. T., Liu, Y., et al. 2012, ApJ, 748, 77, doi: $10.1088 / 0004-637$ X/748/ $2 / 77$

Titov, V. S., Downs, C., Mikić, Z., et al. 2018, ApJ, 852, L21, doi: 10.3847/2041-8213/ aaa3da

Titov, V. S., Downs, C., Török, T., et al. 2021, ApJS, 255, 9, doi: $10.3847 / 1538-4365 /$ abfe0f

Török, T., \& Kliem, B. 2005, ApJ, 630, L97, doi: 10 .1086/462412

Török, T., Panasenco, O., Titov, V. S., et al. 2011, ApJ, 739, L63, doi: $10.1088 / 2041-8205 /$ $739 / 2 / \mathrm{L} 63$

Török, T., Leake, J. E., Titov, V. S., et al. 2018, in AGU Fall Meeting Abstracts, Vol. 2018, SH12A-04

van Ballegooijen, A. A., \& Martens, P. C. H. 1989, ApJ, 343, 971, doi: $10.1086 / 167766$

van Driel-Gesztelyi, L., Baker, D., Török, T., et al. 2014, ApJ, 788, 85, doi: $10.1088 /$ $0004-637 \mathrm{X} / 788 / 1 / 85$

Veronig, A. M., Podladchikova, T., Dissauer, K., et al. 2018, ApJ, 868, 107, doi: $10.3847 /$ $1538-4357$ /aaeac5

Wang, J., Jiang, C., Yuan, D., \& Zou, P. 2021, ApJ, 911, 2, doi: 10.3847/1538-4357/ a.be 637

Wang, J., Simões, P. J. A., \& Fletcher, L. 2018, ApJ, 859, 25, doi: $10.3847 / 1538-4357 /$ a abc0e

Wang, Y. M., \& Muglach, K. 2007, ApJ, 666, 1284, doi: $10.1086 / 520623$ 
Williams, D. R., Török, T., Démoulin, P., van Driel-Gesztelyi, L., \& Kliem, B. 2005, ApJ, 628, L163, doi: $10.1086 / 432910$

Wills-Davey, M. J., \& Thompson, B. J. 1999, Sol. Phys., 190, 467, doi: 10.1023/A: 1005201500675

Zemanová, A., Dudík, J., Aulanier, G., Thalmann, J. K., \& Gömöry, P. 2019, ApJ, 883, 96, doi: $10.3847 / 1538-4357 / a b 3926$

Zhou, T., Ji, H., \& Huang, G. 2008, Adv. Space Res. , 41, 1195, doi: 10.1016/ j.asr.2007. 04.095

Zhou, T.-H., Wang, J.-F., Li, D., et al. 2013, Res. in Astr. and Astrophys., 13, 526, doi: 10 . $1088 / 1674-4527 / 13 / 5 / 004$

Zuccarello, F. P., Aulanier, G., Dudík, J., et al. 2017, ApJ, 837, 115, doi: $10.3847 /$ $1538-4357 / a a 6110$ 